\title{
Large Eddy Simulation study on the structure of turbulent flow in a complex city
}

Article

Accepted Version

Creative Commons: Attribution-Noncommercial-No Derivative Works 4.0

Tolias, I.C., Koutsourakis, N., Hertwig, D., Efthimiou, G.C., Venetsanos, A.G. and Bartzis, J.G. (2018) Large Eddy Simulation study on the structure of turbulent flow in a complex city. Journal of Wind Engineering and Industrial Aerodynamics, 177. 101 - 116. ISSN 0167-6105 doi: https://doi.org/10.1016/j.jweia.2018.03.017 Available at https://centaur.reading.ac.uk/76860/

It is advisable to refer to the publisher's version if you intend to cite from the work. See Guidance on citing.

Published version at: https://www.sciencedirect.com/science/article/pii/S0167610517309765

To link to this article DOI: http://dx.doi.org/10.1016/j.jweia.2018.03.017

Publisher: Elsevier

All outputs in CentAUR are protected by Intellectual Property Rights law, including copyright law. Copyright and IPR is retained by the creators or other copyright holders. Terms and conditions for use of this material are defined in the End User Agreement. 


\section{CentAUR}

Central Archive at the University of Reading

Reading's research outputs online 


\title{
Large Eddy Simulation study on the structure of turbulent flow in a complex city
}

\author{
${\text { I.C. } \text { Tolias }^{\mathrm{a}, 1} \text { • N. Koutsourakis }}^{\mathrm{b}, \mathrm{a}}$ • D. Hertwig • G.C. Efthimiou ${ }^{\mathrm{a}}$ • A.G. Venetsanos ${ }^{\mathrm{a}}$ - J.G. Bartzis ${ }^{\mathrm{b}}$ \\ ${ }^{a}$ Environmental Research Laboratory, INRASTES, National Centre for Scientific Research Demokritos, \\ Patriarchou Grigoriou E \& 27 Neapoleos Str., 15341, Agia Paraskevi, Greece. \\ ${ }^{\mathrm{b}}$ University of Western Macedonia, Dept. of Mechanical Engineering, Sialvera \& Bakola Str., 50100, \\ Kozani, Greece. \\ ${ }^{c}$ Department of Meteorology, University of Reading, Reading, UK.
}

\begin{abstract}
Large Eddy Simulation (LES) of atmospheric flows has become an increasingly popular modelling approach within the last years, as it has the potential to provide deeper insight into unsteady flow phenomena. LES can be improved and validated using specifically designed and well documented wind tunnel datasets. In this work, we evaluate the performance of LES against a wind tunnel experiment in a semi-idealized city ("Michel-Stadt"; CEDVAL-LES database) and use the LES results to study the structure of the turbulent flow at the particular urban area. The first, second and third order statistics are presented, as well as velocity frequency distributions and energy spectra. The results compare well with the experimental values. Information about special features of the flow field is also provided. A particular focus of this work is put on the influence of grid resolution on the results. Five different grids are examined and the required resolution for turbulent flow within the canopy layer is evaluated. This study reveals the strong potential of LES for urban flow simulations. It is shown that LES can assess highly non-Gaussian flow behaviour in street canyons, which has implications for urban ventilation, wind comfort assessment and urban design.
\end{abstract}

Keywords: CFD; Computational Fluid Dynamics; Large Eddy Simulation; Urban turbulent flow; Semiidealized urban canopy; Boundary-layer wind tunnel; Michel-Stadt; LES validation; Grid resolution; ADREA-HF

\section{Introduction}

Within recent years, the physical and geometrical complexity that can be represented with computationally demanding high-resolution, eddy-resolving Computational Fluid Dynamics (CFD) methods has strongly increased. This is markedly the case for studies in urban environments that have become a focal point for both micro-meteorological and wind engineering research communities (e.g. Tamura, 2008; Tominaga and Stathopoulos, 2013; Blocken, 2015). The method of choice here is LargeEddy Simulation (LES), in which the anisotropic energy-carrying eddies in the flow are directly resolved,

\footnotetext{
${ }^{1}$ Corresponding author. Tel: +30 2106503407. E-mail address: tolias@ipta.demokritos.gr (I.C. Tolias).
} 
while the effects of isotropic inertial range and dissipative eddies are parameterized (Piomelli, 2001; Sagaut, 2006; Argyropoulos and Markatos, 2015).

Such turbulence-resolving simulations provide an unprecedented amount of information about the timespace dependent behaviour of flow in the Urban Canopy Layer (UCL), the Roughness Sublayer (RS) and their interaction. For flow in complex urban settings, numerous studies have shown that LES outperforms simulations based on the Reynolds-averaged Navier-Stokes (RANS) equations due to its ability to account realistically for obstacle-induced turbulence (e.g. Murakami, 1998; Xie and Castro, 2006; Tominaga and Stathopoulos 2011; 2012; Gousseau et al., 2011; Nozu and Tamura, 2012). Beyond addressing fundamental research questions, urban LES is now also increasingly used for site-specific studies of pedestrian wind comfort and safety (e.g. Letzel et al., 2012), wind load on buildings (e.g. Nozu et al., 2008, 2015; Elshaer et al., 2016) or urban planning in combination with other CFD approaches (e.g. Yuan et al., 2014; Wang et al., 2017). Another key area where LES is spearheading research progress is urban pollutant dispersion (e.g. Camelli et al., 2006; Xie, 2011; Yoshie et al., 2011; Moon et al., 2014; Michioka et al., 2014; Nakayama et al., 2014; Fuka et al., 2018; Aristodemou et al., 2018). Here LES is used, for example, to deepen the understanding of the unsteady nature of the flow that in turn will lead to better comprehension of the dispersion from continuous or instantaneous releases. Such studies can also contribute to advancements of parameterizations in operational modelling environments (e.g. Giometto et al., 2016) for urban air quality or emergency response dispersion applications (e.g. Patnaik et al., 2012; Leitl et al., 2014, 2016). The time-dependency of the simulation also offers potential to expand deterministic analyses by probabilistic information, e.g. related to occurrence or exceedance probabilities of UCL wind gusts or pollutants concentrations (e.g. Wang et al., 2011; Nakayama and Nagai, 2011; Park et al., 2015; Ikegaya et al., 2017).

Simulation complexity of urban systems is rising due to an increasing level of geometric detail that is covered. Today, the choice to restrict to strongly idealized geometries like uniform buildings arrays is not so much motivated by cost considerations, as by advantages for the study of fundamental turbulence aspects and parameterizations that can be substantiated by a relative abundance of corresponding windtunnel measurements (e.g. Boppana et al., 2010; Gu et al., 2011; Inagaki et al. 2012; Saeedi and Wang, 2015; Goodfriend et al. 2016; Castro et al. 2017; Llaguno-Munitxa et al., 2017). However, in order to investigate the full range of complex geometry-induced flow and dispersion patterns of real cities, studies have to be expanded to realistic, vertically and horizontally heterogeneous canopies (see for example comments on Kanda et al., 2013 and references therein). In such cases, scientific interest is shifting towards simulations based on realistic representations of actual urban areas. Such simulations allow studying the complex interaction of urban turbulence across scales in settings that represent the true heterogeneity of actual street and building layouts. One of the first LES studies of this kind, using the classic Smagorinsky approach as subgrid-scale model and Gaussian turbulence for the inlet of the flow field, was presented by He and Song (1999) in terms of a street-scale study of pedestrian wind comfort between groups of realistic buildings.

More recently, with increasing computational capacities, LES studies of turbulent flow and transport emerged that cover a wide range of built environments scales, from streets and neighbourhoods to entire cities. For example, Nozu et al. (2008) covered an area of $2 \times 1 \mathrm{~km}$ of Tokyo using nested grids in order to estimate the pressure at building walls. Bou-Zeid et al. (2009), examined the effect of the detail of the geometrical representation of a campus on the LES results and concluded that while macroscopically the influence of the details is not important, they can play a significant role when local phenomena between 
the buildings are investigated. They performed the simulation at full-scale and they used RANS-type wall functions with roughness $z_{0}$ equal to $0.01 \mathrm{~m}$ for the building walls and $0.1 \mathrm{~m}$ for the ground. Based on guidelines from previous studies, they used as resolution about 7 cells per obstacle. Xie and Castro (2009), simulated with LES the flow and dispersion over the complex urban geometry of DAPPLE site in London. At the inlet they used the artificial turbulence method of Xie and Castro (2008) that needs as input the integral length scales of turbulence. Those parameters were very roughly estimated at Figure 4 of Xie and Castro (2009), who report that their actual value is not crucial. At the top of the domain they used stress-free boundary, as it is usual at LES studies, even if this boundary condition is not very appropriate for LES.

Liu et al. (2011) modelled with LES part of Macao and compared the results with wind tunnel experimental data. At the inlet they used a simple artificial turbulence method similar to that of Hanna et al. (2002), while at the top of the domain they used non-reflecting boundary condition. Turbulent Prandtl and Schmidt numbers were taken equal to 0.72. Several of such LES studies in cities were reviewed by Gousseau et al. (2011), who also commented on the superiority of LES over RANS methods. Later on, Nozu and Tamura (2012) modeled a part of Tokyo and compared with wind tunnel results. For the inlet they used an auxiliary simulation with a driver region and recycling (Lund et al., 1998; Nozawa and Tamura, 2002). They also performed a RANS simulation and noticed that in that case the pollutant was trapped at the recirculation regions, while in the LES the instability of the flow resulted in intermittent pollution removal out of the recirculation regions, leading to higher dispersion.

In more recent works, Moon et al. (2014) studied the flow in a part of Seoul and found similar results between the classic Smagorinsky and the Vreman (2004) model. The LES simulation of Nakayama et al. (2014) captured the general flow and concentration patterns of a wind-tunnel experiment in a model of Oklahoma city, even if there were some differences compared to the measurements due to the complexity of the case. The inlet conditions were taken from a driver region with a recycling method, the standard Smagorinsky subgrid-scale model with a constant $C_{s}$ value of 0.1 was used and the horizontal grid spacing was about 4 meters. Gousseau et al. (2015) reproduced the wind-tunnel experiments of pollutant dispersion in downtown Montreal using the dynamic Smagorinsky model and the bounded central differences scheme. At the inlet they produced artificial turbulence using the vortex method (Sergent, 2002; Mathey et al., 2006). At the top and lateral boundaries, symmetry boundary conditions were prescribed. They mentioned that the wind flow and dispersion patterns were complex and difficult to predict, even qualitatively. Nozu et al. (2015) applied the standard Smagorinsky model with limited central differences scheme in order to estimate the pressures in a tall building in a real city. They used overset grids, both Cartesian and unstructured, with a grid resolution of $1 \mathrm{~m}$ at their target area. Recently, the coupling of LES in real cities with mesoscale models has also emerged (e.g. Nakayama et al., 2012; Liu et al., 2012; Michioka et al., 2013).

From all the above-mentioned studies of LES in cities, it becomes clear that an accurate prediction and thorough investigation of the flow field is indispensable in order to study pollutant dispersion. Also, so as to examine detailed flow features in complex, realistic city geometries, very detailed and welldocumented experimental data need to be available. Experiments in boundary-layer wind tunnels, like the one examined in the current study, in this regard are an ideal complement to in-situ field measurements as it is possible to measure quantities of interest at a multitude of locations under controlled boundary conditions. 
Very few of the previous urban LES studies considered more than one computational grid and, to the best of our knowledge, they have not examined in detail the actual influence of the grid resolution on the results - something that is common for RANS simulations and easier achievable due to the significantly lower computational costs. Also, few of these studies take advantage of the time-dependent nature of LES, e.g. by performing in-depth analyses of unsteady phenomena in urban flow fields examining higherorder moments like skewness and probability distributions of velocities as in the work presented by Hertwig et al. (2017a, b). In this context, the current work complements the literature by utilizing many computational grids and by examining in detail the flow features emerging in a semi-idealized city for which extensive experimental measurements exist.

The simulated geometry is based on a corresponding wind-tunnel scale model ("Michel-Stadt"; e.g. Hertwig et al. 2012), whose layout is representative for typical central European cities. The experiment was selected because it is a unique case of a semi-idealized city. Moreover, the availability of a vast amount of reference measurements on a dense grid within and above the urban geometry provides an ideal basis for model validation and thorough investigation of the flow field. While some previous LES studies were conducted in the "Michel-Stadt" geometry, presented mainly at conferences (e.g. Koutsourakis et al., 2012; Fuka and Brechler, 2014) and inter-comparison exercises (COST Action ES1006: Gariazzo et al., 2014), they were limited to comparisons of low-order statistics and did not focus on exploring complex flow characteristics in detail, as is the aim of this work.

Here, we assess the performance accuracy of the LES as a function of grid resolution and study local canopy-layer flow features based on an analysis of velocity time series. We examine the general characteristics of the flow at the central part of "Michel-Stadt" with the use of streamtraces and exploit them to better explain local, often unsteady, flow phenomena. This paper also answers a basic question posed in a previous numerical study of "Michel-Stadt" with RANS models (Hertwig et al., 2012): Can we represent the unsteady, time-dependent, structure of turbulent flows and spatial flow patterns faithfully with LES and reproduce, for example, features like bimodal frequency distributions of velocity components that are observed at experimental measurements? Answering this question is of great importance as the field of practical application is shifting its focus to predictions of more specialized variables like extreme values (e.g. for peak concentrations or wind gusts, Efthimiou et al., 2017b; 2017c).

\section{The Michel-Stadt experiment}

The flow data analysed in this study stem from boundary-layer wind-tunnel measurements conducted at the Environmental Wind Tunnel Laboratory (EWTL) of the Meteorological Institute at the University of Hamburg.

The advent and the extensive use of large eddy simulation codes that resolve turbulence directly, created new quality and quantity requirements for experimental measurements used for validation studies. Comparison between LES and experiment can be performed among others with validation metrics, higher-order moments, two-point correlations and other structure recognition methods (Hertwig et al. 2011; 2017a, b). Responding to those needs, the CEDVAL-LES validation database offers time-averaged statistics as well as time-resolved data (usually single-point) for different types of boundary-layer flow 
and dispersion scenarios under neutral stability conditions. The validation datasets are freely available ${ }^{1}$ and described by Fischer et al. (2010) and Bastigkeit (2011).

CEDVAL-LES contains data for various levels of geometric complexity and surface roughness characteristics (e.g. simple rough-wall boundary layer flows above homogenous roughness elements, flows around isolated obstacles or within obstacle arrays, flows in semi-idealized urban environments and in realistic city layouts). The case selected for this study was flow within and above the semi-idealized city geometry referred to as "Michel-Stadt" (case reference: BL3-3).

Fig. 1 presents pictures of the wind-tunnel test section and the urban scale model for this scenario. The semi-idealized urban geometry includes typical features of Northern and Central European cities like courtyards, oblique road arrangements, open spaces and complex intersections. The flow was physically modelled at a scale of 1:225. In the present work, all dimensions are presented in full-scale. The dimensions of the city are $1320 \times 830 \mathrm{~m}$ (length $\mathrm{x}$ width). Three building heights are included in the model: 15, 18 and $24 \mathrm{~m}$ (Fig. 2). In the setup investigated here, all buildings have flat roofs.

The BL3-0 experimental case of the CEDVAL-LES database was designed in order to correspond to the approach flow section upwind of the buildings of the BL3-3 case and has the same roughness length $z_{0}$ as that of the city $(1.53 \mathrm{~m})$. Thus, the setup of the very rough boundary-layer flow (BL3-0) was used in this study as the inflow boundary layer for the semi-idealized city case (BL3-3).
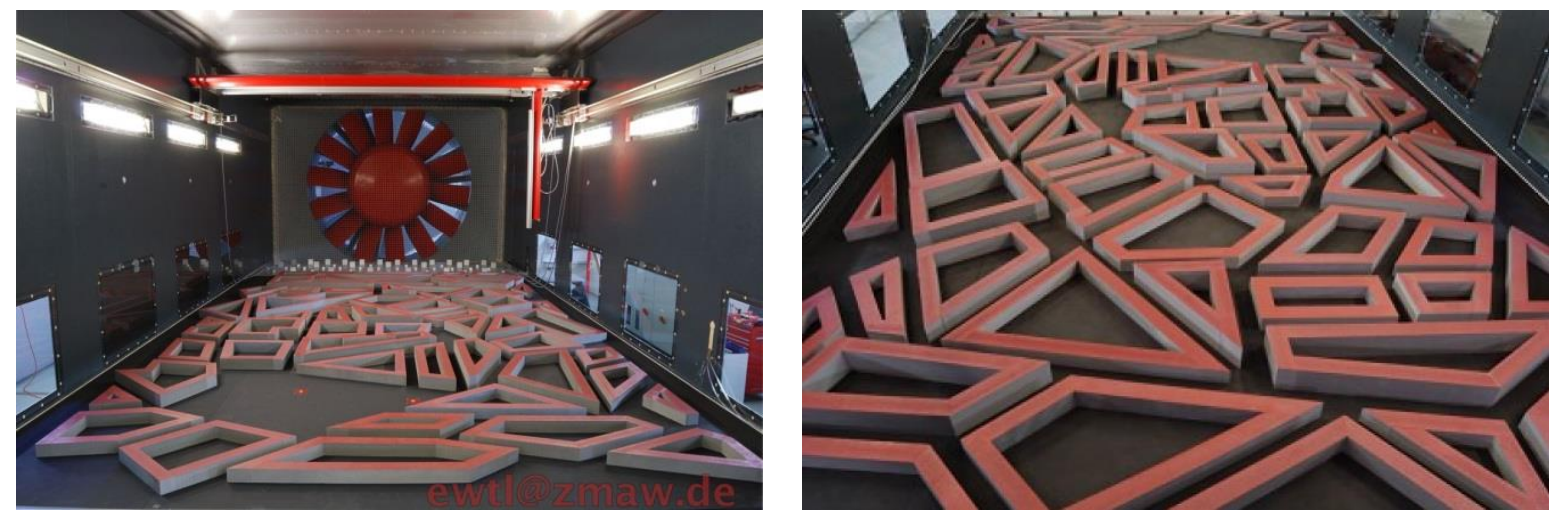

Fig. 1: The semi-idealized urban geometry used in this study (picture courtesy of EWTL, Bernd Leitl left picture is from a later setup that had roughness elements both before and after the city).

Velocity measurements were conducted with Laser Doppler Anemometry (LDA), providing point-wise information about the horizontal velocity components within and above the city. The vertical velocity was not measured. The streamwise reference velocity at a height of $100 \mathrm{~m}$ was kept close to $U_{r e f}=6.1 \mathrm{~m} / \mathrm{s}$ and was monitored and recorded during each measurement run. The reference velocity was verified to be sufficiently high to guarantee Reynolds number independence of derived flow statistics within and above the urban canopy. Three groups of wind speed measurements were used in this study: 1) Detailed measurements on horizontal cross-sections at heights of 2, 9 and $18.23 \mathrm{~m}$ in the model centre (340 $\mathrm{m} \mathrm{x}$ $340 \mathrm{~m}$ core domain). These measurements covered various street canyons, a large open space and 2 courtyards with overall 383 measurement points per level (Fig. 2, left). 2) 40 vertical profiles distributed at various points throughout the city (Fig. 2, centre) in order to create a general view of the flow. 3) Measurements on at horizontal cross-sections above the canopy layer at heights of 27.45 and $30.15 \mathrm{~m}$,

\footnotetext{
${ }^{1}$ https://mi-pub.cen.uni-hamburg.de/index.php?id=6339 (last assessed 30/03/18)
} 
containing 252 data points at each height (Fig. 2, right). More details about this experiment can be found in Hertwig et al. (2012).

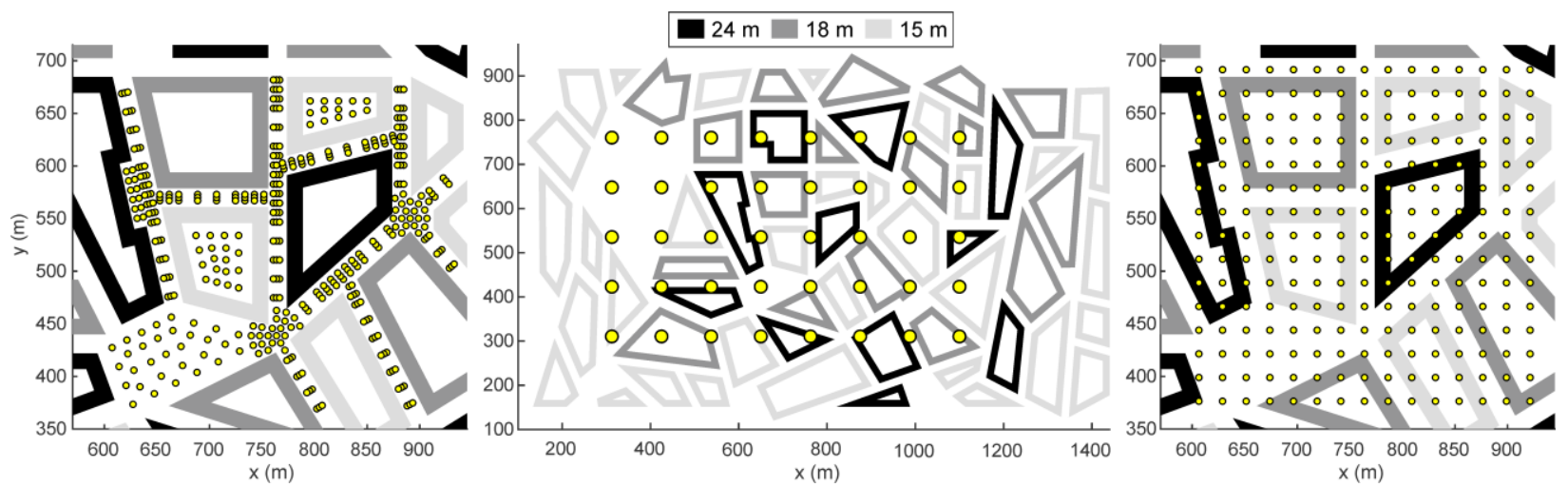

Fig. 2: Top views of the city (whole city in the middle) with an indication of buildings height and measurements location. Left: densely-spaced measurement locations within the urban canopy layer at elevations of 2, 9 and $18.23 \mathrm{~m}$; centre: vertical profiles locations; right: measurements locations above roof top at heights of 27.45 and $30.15 \mathrm{~m}$.

\section{Numerical methodology}

\subsection{Governing equations}

In LES, a spatial filtering is applied to every flow variable, decomposing it into a resolved (or filtered) component and a Sub-Grid Scale (SGS) component. The filtered governing equations that are solved are:

$$
\begin{gathered}
\frac{\partial \bar{\rho}}{\partial t}+\frac{\partial\left(\bar{\rho} \vartheta_{\rho}\right)}{\partial x_{j}}=0 \\
\frac{\partial\left(\bar{\rho} \vartheta_{i}\right)}{\partial t}+\frac{\partial\left(\bar{\rho} q_{i} q_{j}\right)}{\partial x_{j}}=-\frac{\partial \bar{p}}{\partial x_{i}}+\frac{\partial\left(\epsilon_{i j}+\tau_{i j}^{R}\right)}{\partial x_{j}} \\
\bar{p}=\bar{\rho} r \bar{T}
\end{gathered}
$$

where $\rho$ is the density, $t$ is the time, $u_{j}$ are the velocity components, $x_{j}$ is the distance, $p$ is the pressure, $r$ is the gas constant and $T$ is the absolute temperature. The bar represents space-averaged values, while the tilde denotes density-weighted space-averaged values. $t_{i j}^{\prime}$ is the instantaneous shear stress tensor due to molecular forcing and $\tau_{i j}^{R}$ is the residual stress tensor due to the subgrid turbulence. In this study, the SGS stresses are modelled using the Smagorinsky (1963) subgrid scale model with the Van Driest (1956) damping in order to account for the reduced growth of the small scales near the wall:

$$
\begin{gathered}
\tau_{i j}^{W_{i}}=2 \mu \mathcal{S}_{i j}^{\circ}-\frac{2}{3} \mu \frac{\partial \vartheta_{k}}{\partial x_{k}} \delta_{i j} \\
\tau_{i j}^{R}=2 \mu_{t} \xi_{i j}^{o}-\frac{1}{3} \tau_{k k}^{R} \delta_{i j}
\end{gathered}
$$




$$
\begin{gathered}
\mu_{t}=\bar{\rho}\left[C_{s} \Delta\left(1-e^{-y^{+} / 25}\right)\right]^{2} \sqrt{2 S_{i j}^{\delta} S_{i j}^{O}} \\
S_{i j}=\frac{1}{2}\left(\frac{\partial \psi_{i} o}{\partial x_{j}}+\frac{\partial \psi_{j}}{\partial x_{i}}\right)
\end{gathered}
$$

where $\mu$ is the dynamic viscosity, $S_{i j}$ is the rate-of-strain tensor, $\delta_{i j}$ is the Kronecker delta, $C_{s}$ the Smagorinsky constant, $\Delta$ is the filter size and $y^{+}$is the non-dimensional distance from the wall. The Smagorinsky constant was set equal to 0.1 and the filter size $\Delta$ equal to $V^{1 / 3}$ where $V$ is the volume of the

computational cell. The term $\frac{1}{3} \tau_{k k}^{R} \delta_{i j}$, which is usually negligible compared to thermodynamic pressure (Erlebacher et al., 1992), is incorporated into the filtered pressure. The effect of gravity was ignored in order to better monitor small pressure changes in the flow field.

\subsection{Simulation setup}

The city was considered in full scale; thus, the values of all variables are given in full scale in the rest of the text. The computational domain size is $1670 \times 900 \times 147 \mathrm{~m}$ in the streamwise (x axis, $u$ velocity component), spanwise (y axis, $v$ velocity component) and vertical ( $\mathrm{z}$ axis, $w$ velocity component) directions, respectively. The coordinate system has its origin at the point $(-765.05 \mathrm{~m},-532.9 \mathrm{~m}, 0 \mathrm{~m})$ of the coordinate system of the experiment. The boundaries of the domain are shown in Fig. 3. The distance of the west boundary (left side of the domain, Fig. 3) from the first obstacle is equal to $120 \mathrm{~m}$ (i.e. $8 \mathrm{H}$ where $\mathrm{H}=15 \mathrm{~m}$ is the height of the buildings in the first and last row) and the distance of the east boundary from the last obstacle is equal to $230 \mathrm{~m}(15 \mathrm{H})$. The height of the domain corresponds to 6.1 times the maximum height of the buildings. These dimensions agree with recommendations for microscale CFD modelling of urban flows defined in the COST Action 732 (Schatzmann et al., 2010). The distance of the lateral boundaries from the nearest obstacle is approximately $35 \mathrm{~m}$ which is about the distance (in full scale) between the wind-tunnel walls and the nearest building. As a test, a larger domain length was also used in order to examine the impact on the results. In that case, the inlet plane was moved $1500 \mathrm{~m}$ upwind of the city and a series of roughness elements (24 rows of obstacles based on floor roughness dimensions used in the experiment) were positioned in the area in front of the city. No significant changes in the results at the sensors' positions were observed (see also Sect. 4.2). 


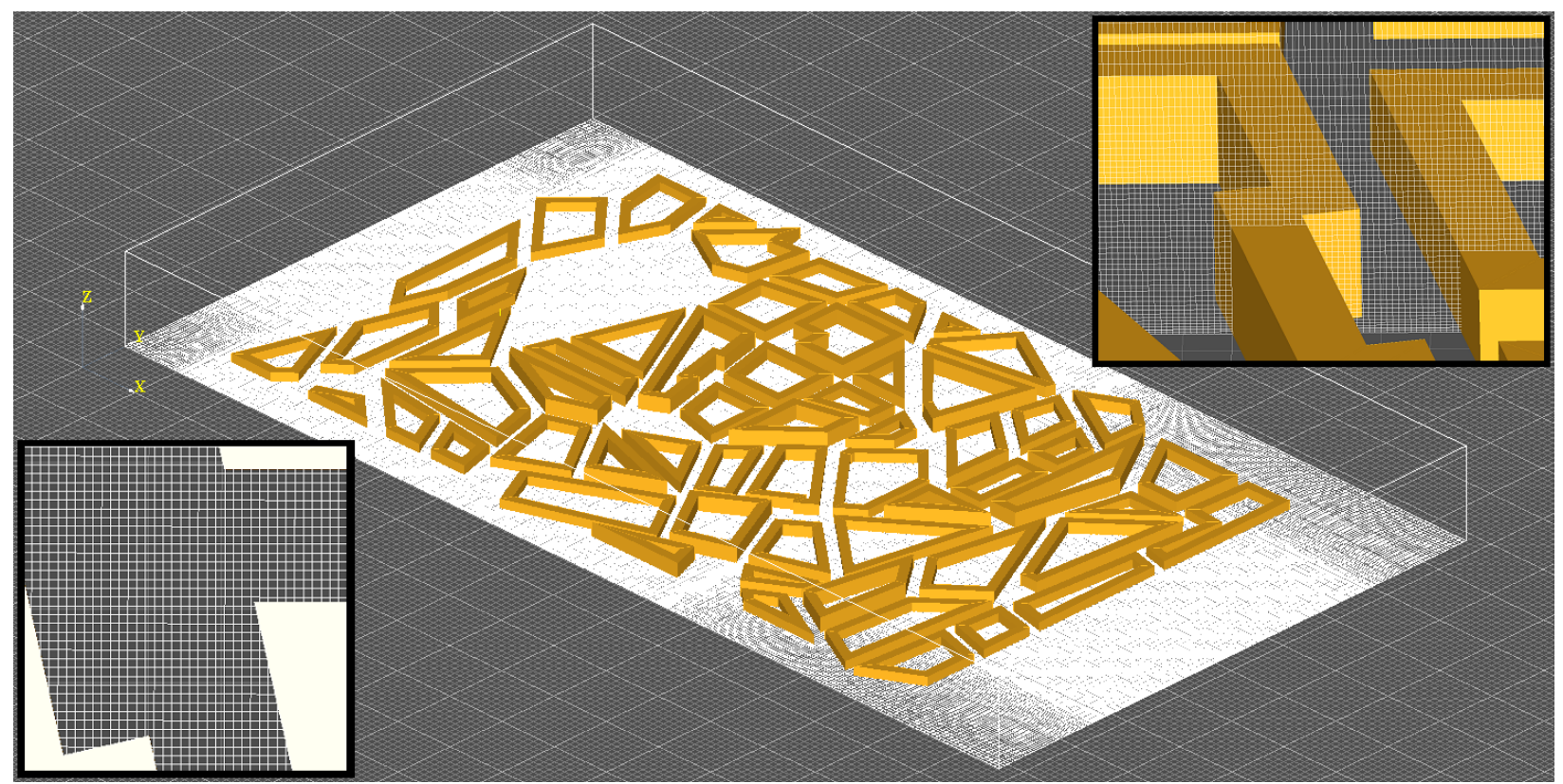

Fig. 3: The size of the computational domain used in the simulations along with near-wall details of Grid 5 shown in the insets.

Five grids were used for the discretization of the domain in order to study the effect of numerical resolution on the accuracy of the resulting flow fields. Their main characteristics are presented in Table 1. The ratio between the number of cells of successive grids ranges between 4 for the coarsest grids and 1.9 for the finest grids. For Grids 3-5, the height of the cells near the ground satisfies the minimum grid resolution proposed by Tominaga et al. (2008), i.e. 1/10 of the maximum building height of $24 \mathrm{~m}$. For Grids 2-4 the vertical grid resolution is constant from the ground up to $27 \mathrm{~m}$ (thus the core domain of the city has cubic cells) and then continuously stretched with an expansion factor of 1.06. In Grid 5 the resolution is increased from the ground to $9.6 \mathrm{~m}$ from 0.5 to $1.2 \mathrm{~m}$ and then it is kept constant until $36 \mathrm{~m}$. Above this height, it is vertically expanded by a factor of 1.07. In the horizontal directions $\mathrm{dx}$ and dy are kept constant in the core of the building domain spanning an area of $825 \mathrm{~m}$ times $436 \mathrm{~m}$. Outside the core region, for Grids 2-5 dy is expanded with a factor of 1.02, while $\mathrm{dx}$ is expanded with a factor of 1.06 and 1.01 in upwind and downwind directions, respectively. The structure of Grid 5 is shown in Fig. 3 in terms of xy and xz planes.

\begin{tabular}{|c|c|c|c|c|}
\hline Grid & Number of cells & $\begin{array}{c}\text { Number of cells ratio } \\
(\text { Grid } \boldsymbol{i} / \text { Grid } \boldsymbol{i}-\mathbf{1})\end{array}$ & $\begin{array}{c}\boldsymbol{d} \boldsymbol{x}, \boldsymbol{d y} \mathbf{( m )} \text { at the core of } \\
\text { the urban area }\end{array}$ & $\begin{array}{c}\text { Minimum } \boldsymbol{d} \boldsymbol{z} \\
\text { (ground level) }\end{array}$ \\
\hline Grid 1 & 718,181 & - & 6.6 & 3.2 \\
\hline Grid 2 & $2,863,432$ & 4 & 3.0 & 3.0 \\
\hline Grid 3 & $7,448,672$ & 2.6 & 2.0 & 2.0 \\
\hline Grid 4 & $14,707,038$ & 1.97 & 1.5 & 1.5 \\
\hline Grid 5 & $28,114,306$ & 1.9 & 1.2 & 0.5 \\
\hline
\end{tabular}

Table 1: Characteristics of the examined grids.

At the lateral boundaries, solid surfaces were considered as boundary conditions to mimic the situation in the wind tunnel. This specification gives the same results, but with a slight acceleration of the mean flow, compared to the case of using symmetry boundary conditions at the lateral planes instead of solid walls, 
which were also tested. This acceleration was taken into account before processing the results in the estimation of the reference velocity for the predictions, which is defined at a height of $100 \mathrm{~m}$ as in the experiment. The reference velocity was estimated to be equal to $U_{r e f}=6.84 \mathrm{~m} / \mathrm{s}$ for the dense grids.

At the outflow plane a non-reflecting type boundary condition for the normal velocity component and a zero-gradient boundary condition for the other velocity components were used. Rough-wall wall functions were used at the building surfaces and the ground with a roughness length $z_{0}$ equal to $0.0625 \mathrm{~m}$. At the area in front of the city a higher roughness length was used $(1.498 \mathrm{~m})$ for the coarse Grids 1 and 2, while for the finer grids that roughness was set equal to that of the ground inside the city.. At the inlet and the top boundary, a given value equal to zero was used as boundary condition for the $v$ and $w$ velocity components whereas the Langevin-type boundary condition was used for the $u$ velocity component (details are given in the following section). Finally, as initial conditions, the vertical velocity profile of the mean flow that was imposed in the inlet was used in the whole domain.

\subsubsection{Langevin-type inflow boundary condition}

In LES suitable boundary conditions are required especially at the inlet planes in order to reproduce realistic turbulent flow conditions and to minimize the time/space that is needed from the code to develop the turbulent flow field. A common approach, particularly for simulations in regular building arrays, is to use cyclic boundary conditions. This method has some disadvantages, the most significant of which being that it cannot be applied to any arbitrary geometry. Many alternative methods have been proposed which can be broadly split into two categories. The first type uses explicitly or implicitly a method to 'recycle' the flow, frequently in a driver region that exists separately to the actual domain of interest. Consequently, some of the disadvantages of using cyclic boundary conditions remain. An example of this approach is described by Lund et al. (1998). In the second type, artificial turbulence is generated based on statistical characteristics of the flow (e.g. Smirnov et al. 2001; Klein et al. 2003, Xie \& Castro 2008) or by defining characteristics of turbulence structures (e.g. Jarrin et al. 2006). Recent reviews on inflow turbulence generation methods are presented by Tabor and Baba-Ahmadi (2010) and Wu (2017).

In the present work, the second type of inlet boundary conditions was utilized. The Langevin-type equations (Koutsourakis, 2014) were used which can provide coherent fluctuations at any free boundary of the computational domain. In this method, the velocity component $u_{i}$ is analysed as the sum of a mean value $\bar{u}_{i}$ and a fluctuation $u_{i}^{\prime}$, i.e. $u_{i}=\bar{u}_{i}+u_{i}^{\prime}$. If the fluctuation at some boundary cell (at which the Langevin-type boundary condition is applied) at time $t$ is equal to $u_{i}^{\prime}(t)$, then the fluctuation at $t+\Delta t$ is given by:

$$
u_{i}^{\prime}(t+\Delta t)=\left(1-\frac{\Delta t}{T_{u_{i}}}\right) u_{i}^{\prime}(t)+\sigma_{u_{i}}\left(\frac{2 \Delta t}{T_{u_{i}}}\right)^{1 / 2} \xi
$$

where $T_{u_{i}}$ is the autocorrelation time scale, $\sigma_{u_{i}}$ is the standard deviation and $\xi$ is a random number that follows the Gaussian distribution with a mean equal to 0 and standard deviation of 1 . The above equation correlates the fluctuations in each cell with the fluctuation in the same cell at the previous time-step. The first term of this equation represents the dependence of $u_{i}^{\prime}$ on its previous value and the second term the random part of $u_{i}^{\prime}$. A similar approach was used by Hanna et al. (2002). The above equation is derived 
from the equation $u_{i}^{\prime}(t+\Delta t)=a u_{i}^{\prime}(t)+\beta \xi$ with the autocorrelation function of $u_{i}^{\prime}$ being given by the relation $R_{u_{i}}(\Delta t)=e^{-\Delta t / T_{u_{i}}} \approx 1-\frac{\Delta t}{T_{u_{i}}}$.

In contrast to the mere Gaussian fluctuations, the Langevin-type method can provide enough coherence for the turbulence not to be damped, while still remaining simple. However, as formulated above, it does not provide the spatial correlation of $u_{i}^{\prime}$ among neighbouring cells. An extension of this formula that considers spatial correlations has also been developed and tested (Koutsourakis, 2014). From those tests it was found that the spatial correlations are of secondary importance compared to temporal correlations concerning inflow turbulence generation for LES (see also Davidson, 2005). Also, according to our experience, the LES model will produce its "own turbulence", that could partially deviate from the exact inlet characteristics. As a result, a simple inlet condition which triggers the code to generate and maintain a turbulent flow field seems to be sufficient for general purpose LES calculations around buildings (see also Davidson, 2005 and Tabor and Baba-Ahmadi, 2010). Thus, equation Error! Reference source not found. was used in the present study, which provides only time correlation of the imposed velocity fluctuations at the inlet.

The Langevin-type boundary condition was initially tested in the case of the horizontally homogeneous rough boundary-layer flow without buildings (BL3-0) at wind tunnel scale (Koutsourakis, 2014). It yielded better results than the cyclic conditions, mainly due to its application to the top boundary, since the symmetry-on-top of the cyclic case dampens turbulence. Indeed, in a simulation with cyclic conditions in longitudinal direction and Langevin-type on top, the results were the same as in the case of Langevin-type at inlet and top. This is a confirmation that the Langevin-type boundary condition works well. One of its advantages is that it offers the ability to perform LES in any heterogeneous building geometry (where cyclic conditions might be impossible to apply) and is also a good boundary condition for the free top surface, even if cyclic or any other type of boundary conditions are used elsewhere.

In the current simulations, the Langevin-type boundary condition was used for the $u$ component of the velocity at the inlet and the top boundaries of the domain. To be applied, it is necessary to provide at each cell the mean values, the standard deviation and the autocorrelation time scale. These values were derived from the BL3-0 measurements as a function of height. Thus the imposed inlet profile corresponds to the conditions modelled in the wind tunnel approach flow section upwind of the urban model. The mean values of $u$ (for Grid 5) were between $0.061 \mathrm{~m} / \mathrm{s}$ (on the ground cell) and $6.71 \mathrm{~m} / \mathrm{s}$ (at the top cell). The value of $T_{u_{i}}$ at the ground cell was equal to $45 \mathrm{~s}$, increasing to the value of $198 \mathrm{~s}$ at $53.7 \mathrm{~m}$ and then decreasing to $153 \mathrm{~s}$ at the top cell. The initial values of $u_{i}^{\prime}$ at the ground cell was equal $6.6 \mathrm{e}-05 \mathrm{~m} / \mathrm{s}$, increasing to the value of $1.45 \mathrm{~m} / \mathrm{s}$ at $30.6 \mathrm{~m}$ and then decreasing to $0.9 \mathrm{~m} / \mathrm{s}$ at the top cell.

\subsection{Numerical details}

The CFD code ADREA-HF was used for all simulations in this study. ADREA-HF is a general purpose CFD code which has been extensively validated against flow and dispersion experiments (Venetsanos et al. 2010, Hertwig et al. 2012, Efthimiou et al. 2017a, 2017b). It uses a finite volume method on a staggered Cartesian grid. Any solid surface is represented with high accuracy, without 'staircase' effects, with the use of porosities. Based on the porosity method (Bartzis, 1991) the cells can be entirely blocked, partially blocked or entirely free. The fully blocked cells (e.g. within buildings) are excluded from the 
calculations and memory allocations, while for the partially blocked cells the integration of the conservation equations is performed over the active (free) cell volume, taking into account the position and the orientation of all in-cell surfaces. Face areas of the control volumes are treated similarly.

The pressure and velocity equations are decoupled using a modification of the SIMPLER algorithm (Kovalets et al., 2008). For the discretization of the convective terms in the momentum equations a second order accurate bounded central difference scheme was used (Waterson and Deconinck, 2007). The implementation was carried out using a deferred-correction approach via the source term. For the time advancement, an implicit second order backward differences scheme was chosen. The time step $\Delta \mathrm{t}$ was set equal to $0.2 \mathrm{~s}$ for the Grid 1 and Grid 2 cases and equal to $0.1 \mathrm{~s}$ for the higher resolution grids in order for the Courant-Friedrichs-Lewy (CFL) number to be smaller than 1 in all cases and to ensure proper time resolution for turbulence and fast convergence. The values 0.1 and $0.2 \mathrm{~s}$ correspond to non-dimensional time steps $\Delta t^{*}=\Delta t \cdot U_{\bar{H}} / \bar{H}$ of 0.02 and 0.04 (where $\bar{H}$ is the mean building height, equal to $19 \mathrm{~m}$. and $U_{\bar{H}}$ the inflow velocity at this height, equal to $3.85 \mathrm{~m} / \mathrm{s}$ ). In the case of Grid 5 , the CFL number was approximately equal to 0.8 and 4-5 iterations were required for convergence in each time-step (maximum absolute error for velocities equal to about $0.003 \mathrm{~m} / \mathrm{s}$ ). Smaller time steps, equal to 0.02 and $0.04 \mathrm{~s}$ were also tested in Grid 3 and the results were found to be independent of the time-step.

The computations were run in parallel using the Greek National High Performance Cluster (HPC) ARIS (consisting of 426 nodes, two Intel Xeon E5-2680 v2 processors and 64 GB of RAM per node, Infiniband FDR network). For the parallelization of the code the domain decomposition method is used. The computational domain is split into sub-domains (partitions) and each sub-domain is assigned to a processor core. The communication between the cores is achieved using the MPI protocol. The linear system for the pressure that arises from the discretization of the differential equations is solved using the BiCGstab method with ILU(0) preconditioning (Saad, 2003). A block variation of the ILU preconditioner is used which is easy to be parallelized. Each core applies the ILU preconditioner only for the data of the assigned sub-domain. As a result, each core has its own precondition matrix and all precondition operations can be carried out in parallel. The average number of iterations for the convergence of the BiCGstab method was equal to 58 and 68 for Grid 4 and 5 respectively. The code exhibited very good speed-up as the number of cores increased. For the case of Grid 4 the speedup of 3.8 was achieved by increasing the number of cores by a factor of four (from 60 to 240). The CPU time for the whole simulation was 22 days for Grid 4 using 100 cores and 35 days for Grid 5 using 170 cores.

Velocity time series were extracted at overall 2,158 locations throughout the domain, corresponding to the measurement sites of the experiment. A sampling time interval equal to $1.0 \mathrm{~s}$ was usually chosen (nondimensional value equal to 0.2). Smaller sampling rates did not reveal any change of the time-series statistics. The simulated time was set equal to $10,000 \mathrm{~s}$ which corresponds to approximately 40 times the bulk eddy turnover time $L / U_{\text {ref }}$, where $L$ is the domain length. Such a long modelling time is of particular importance when analysing higher-order statistics as done in this study. It is noted that in order to compare the numerical and experimental results, it should be taken into account that due to the length scale reduction in the wind tunnel by a factor of 225, processes happen 225 times faster at the same wind speed as under full scale conditions. For that reason statistics like the spectra presented later on, are compared in dimensionless form. 
After a simulation time of $1,000 \mathrm{~s}$ (4 times the bulk eddy turnover time), the turbulent flow field had fully developed. The sensor time-series in the period of 1,000 to $10,000 \mathrm{~s}$ were used in order to obtain the statistics at the 2,158 points. Moreover, mean values and turbulent stresses are calculated at all grid points and stored at chosen times by performing averaging during the simulation.

\subsection{Statistical Performance Measures}

Statistical Performance Measures (SPMs) provide a quantitative means of comparison between model predictions and measurements. SPMs are very useful for model evaluation especially in case of a large number of data pairs to compare. Several SPMs are available and each of them has its merits and drawbacks (Schatzmann et al., 2010). In the present study, two SPMs are used: Hit Rate (HR) and FAC2.

The use of the Hit Rate for the evaluation of micro-scale models was proposed by COST Action 732 (Schatzmann et al., 2010). In principle, the Hit Rate specifies the fraction of predictions that differ from the observations within an allowed range given by the relative error $D$. The experimental uncertainty is taken into account with the parameter $W$, which expresses a "low value" limit. HR is then obtained from:

$$
H R=\frac{1}{n} \sum_{i=1}^{n} N_{i}, \quad N_{i}= \begin{cases}1, & \text { if }\left|\frac{P_{i}-O_{i}}{O_{i}}\right| \leq D \text { or }\left|P_{i}-O_{i}\right| \leq W \\ 0, & \text { else }\end{cases}
$$

where $n$ is the total number of data pairs, $P$ is the model's prediction and $O$ is the corresponding experimental observation. The minimum value of $\mathrm{HR}$ is 0 and its maximum equal to 1 , which represents a perfect agreement with the experiment. The parameter $W$ was set to 0.0165 and 0.0288 for the $\bar{u} / U_{\text {ref }}$ and $\bar{v} / U_{\text {ref }}$ normalized mean velocity components, respectively. These values are based on the statistical scatter of the "Michel-Stadt" experiment that was derived from repetition measurements (Hertwig et al., 2012). The parameter $D$ was selected equal to 0.25 following recommendations in VDI (2005).

The FAC2 metric gives the fraction of predictions that are within a factor of 2 of the observations:

$$
F A C 2=\frac{1}{n} \sum_{i=1}^{n} N_{i}, \quad N_{i}= \begin{cases}1, & \text { if } 0.5 \leq \frac{P_{i}}{O_{i}} \leq 2.0 \text { or }\left(O_{i} \leq W \& P_{i} \leq W\right) \\ 0, & \text { else }\end{cases}
$$

FAC2 takes values between 0 and 1. A perfect model would have FAC2 $=1$. A "low value" limit is also included in this metric through the parameter $W$, which values are the same as for the Hit Rate.

\section{Results and discussion}

\subsection{General flow features and grid sensitivity assessment}

Fig. 4 presents vertical profiles (profile 14 and 37) of the normalized mean streamwise $\bar{u} / U_{\text {ref }}$ and lateral $\bar{v} / U_{\text {ref }}$ velocity components at two different locations of the urban area. Profiles positions are indicated in figure insets. Both points are situated on the centreline of the town, the former in the first upwind part of the city and the latter in the centre. The flow behaviour is quite different because of the different local 
building layout. Moreover, these selected profiles are representative of the profiles in the rest of the city in terms of the local flow regimes and the grid effect on the simulation results.

Regarding the streamwise velocity component (Fig. 4, top), the effect of the geometry on the profiles is obvious. In Profile 37, which is located inside a narrow street canyon, near zero values of the streamwise velocity are observed from the ground until $20 \mathrm{~m}$. On the other hand in Profile 14, which is located at the entrance to a larger open area, velocity values are negative near the ground and then gradually increase with height. The LES and the experiments show the same qualitative trends although LES has a tendency to underestimate the velocities. The underestimations are mainly due to, or correlate to, the more severe underestimation of the stresses (which are presented in the next section) at these points. In general, the finer the grid, the closer the results are to the experimental values, even if the differences are very small. Results from Grids 4 and 5 overlap especially at profile 37. It is interesting that, even if we refer to mean values, the profiles of both the experiment and the simulations may not be smooth due to the unsteadiness of the flow (see for example profile 14 at $60-80 \mathrm{~m}$ ).

Regarding the lateral velocity component (Fig. 4, bottom), a strong amplification within the canopy layer is observed because of building-induced flow effects. Here, the impact of the grid resolution on the computational results is particularly striking. In profile 14, the two coarser grids (Grid 1 and 2) fail to reproduce the overall shape of the experimental curve since a positive sign of the $\bar{v}$ velocity is predicted at all heights. On the other hand, the denser grids (Grid 3 to 5) predict the negative sign of the velocity at heights lower than about $20 \mathrm{~m}$. The reason for this is a recirculation that formed in the area, which cannot be reproduced accurately on the coarse grids. As the number of cells increases, the agreement with the experiment is strongly improved. However, significant under-prediction exists even for Grid 5 at heights below $10 \mathrm{~m}$. For the narrow street canyon location (Profile 37), considerable qualitative and quantitative differences can be observed among the computational results. Due to the strong geometric confinement of the flow, the detailed flow structure cannot be resolved with the coarser grid and the results show a very high sensitivity to the choice of the grid. At heights below the mean building height, only Grid 4 and Grid 5 predict the negative velocities that are observed in the experiment. However, even though Grid 5 is in better agreement with the experiment, it does not capture all oscillations of the profile. 

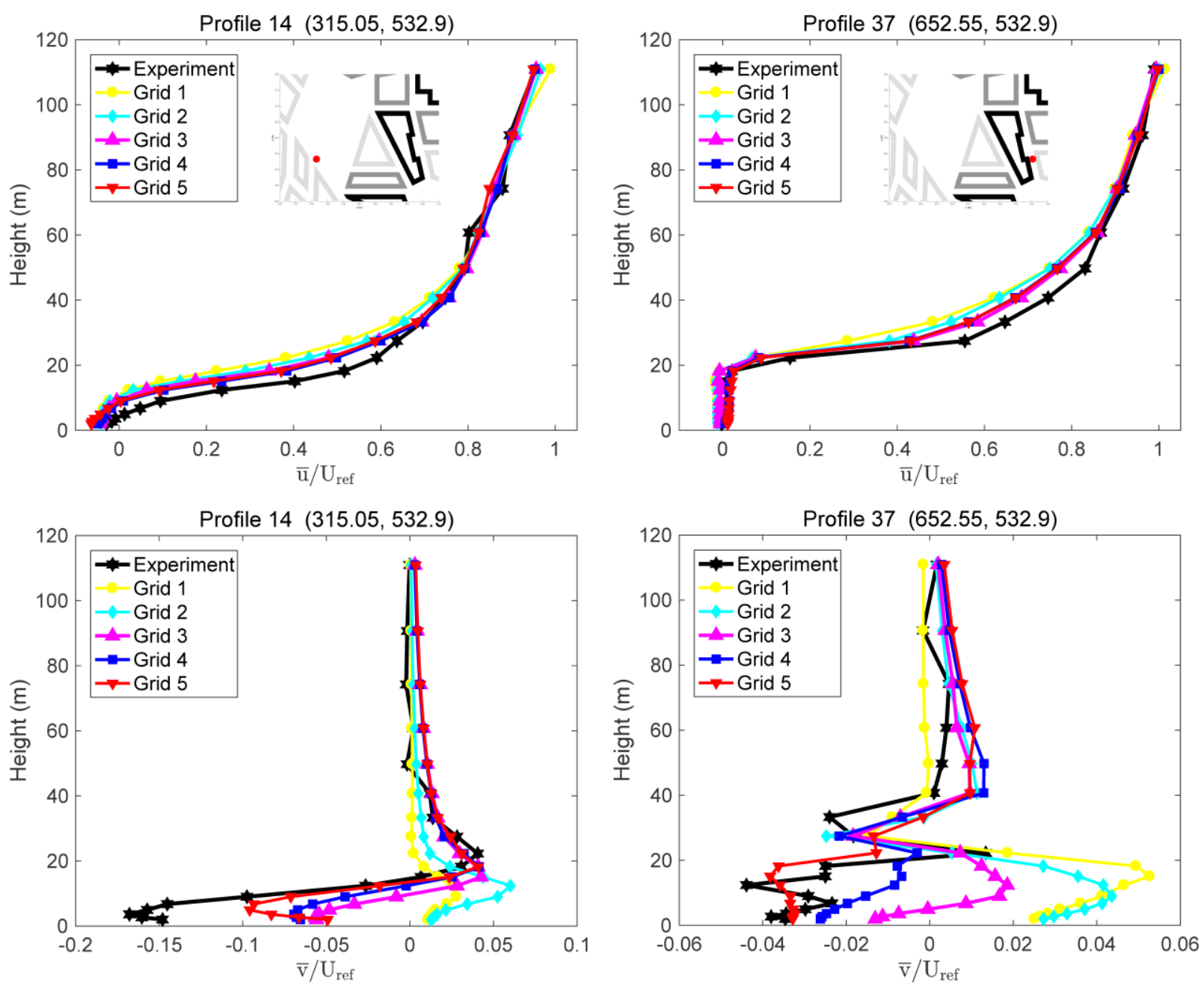

Fig. 4: Vertical profiles of the normalized mean streamwise, $\bar{u} / U_{\text {ref }}$, and lateral, $\bar{v} / U_{\text {ref }}$, velocity components at two representative locations of the urban area. The position of the profiles in the city is marked with a red dot in the insets.

In Fig. 5 the scatter plots of the normalized mean velocities for Grid 2 (coarse grid) and Grid 5 (finest grid) are presented (videos of the full evolution of the scatter plots as the grid is refined are presented as supplementary materials. Supplementary video related to this article can be found at https://doi.org/10.1016/j.jweia.2018.03.017). The results have been grouped based on height ranges. We observe that most of the points fall within the limits of the "1 to 2 " and " 2 to 1 " lines for both grids. However, the grid choice has a significant impact on the model performance. The overall scatter of the values about the 1-to-1 line decreases for the finer grid, which is most obvious in the lateral component especially at low magnitudes. The alignment of the results with the ideal line for the fine grid is also improved. This improvement is particularly obvious for the lowest comparison heights within the canopy layer. However, for both grids the LES has a tendency to underestimate the canopy layer velocities ( $\mathrm{z}<28$ $\mathrm{m})$ compared to the experiment. The underestimation, in absolute values, becomes higher as we move closer to the ground and it is lower for the finer grid. The use of wall functions may be partly responsible for this. Wall functions always influence the accuracy of the simulation, especially when the exact value of the roughness is not known. Close to the ground, especially in the region between the ground and $7 \mathrm{~m}$, 
a larger fraction of negative velocities are observed due to the flow field complexity (e.g. recirculation zones). Values of the mean vertical velocity $\bar{w}$ cannot be evaluated due to the lack of experimental measurements.
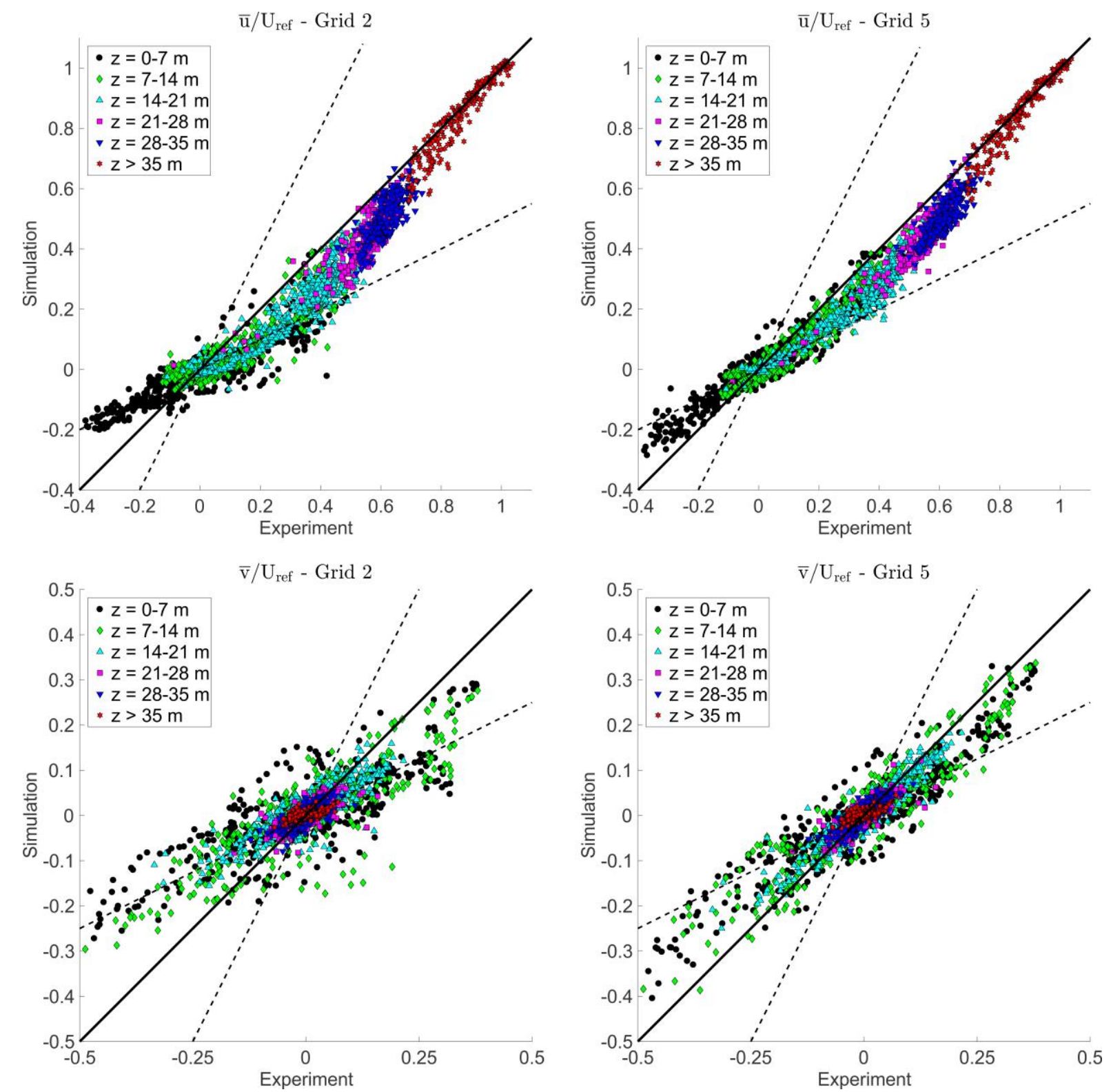

Fig. 5: Scatter plots of the normalized mean streamwise $\bar{u} / U_{\text {ref }}$ (top) and lateral $\bar{v} / U_{\text {ref }}$ (bottom) velocity components for Grid 2 (left) and Grid 5 (right) showing all 2,158 data pairs grouped by different height ranges. The dashed lines indicate the 1-to-2 and 2-to-1 margins.

In a next step, these results are quantified by analysing the evolution of validation metrics as a function of grid resolution. Fig. 6 presents the two validation metrics introduced before for all five grids used in this study. As indicated in the scatter plots, for both velocity components the performance of the model increases with increasing grid resolution. The largest differences between simulations and experiment are observed for the two coarsest grids (Grid 1 and 2). The differences between the finest grids are low and in some cases the curves flatten out quite quickly into plateaus, i.e. the gain in accuracy from refining the 
grid further becomes negligible. This result is particularly important as further grid refinements are associated with a strong increase of overall computational costs. Taking into account that the main difference between Grid 4 and Grid 5 is the number of cells in the vertical direction, we notice that a resolution of about 10 cells through the average building height is in general sufficient to resolve the basic flow characteristics in LES. This result is in accordance with recommendations by Tominaga et al. (2008).

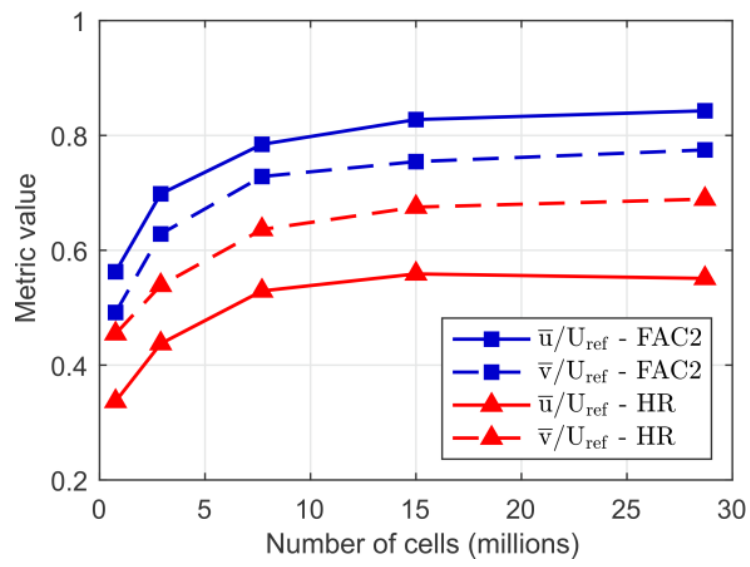

Fig. 6: Validation metrics for the normalized mean velocity components $\bar{u} / U_{\text {ref }}$ and $\bar{v} / U_{\text {ref }}$ for all LES grids examined.

The energy spectra of the $u$ velocity component at a single point for all grids are displayed in Fig. 7. The specific position represents a point above the courtyard of the $15 \mathrm{~m}$ high building D (see Fig. 8). In that area the flow is influenced by the buildings, but it is not too complex; also, for this point, the comparison between the experimental values and the LES results is satisfactory compared to other positions (predicted to experimental ratio of $\bar{u} / U_{\text {ref }}, \bar{v} / U_{\text {ref }}, \sigma_{u} / U_{\text {ref }}$ and $\sigma_{v} / U_{\text {ref }}$ equal to $0.72,0.96,0.80$ and 0.69 respectively). In the same figure, the experimental spectrum is also displaced. We observe that all LES spectra have a good agreement with the experiment up to the spectral peak. After the maximum value is reached, the spectra of Grids 2 to 5 agree well with the experiment in the starting region of the inertial subrange, following the expected $-2 / 3$ law (scaled frequencies). This means that part of the inertial subrange of turbulence is resolved, indicating a proper LES method for the chosen grid resolutions. The absence of the inertial subrange from the spectrum of Grid 1 implies that this grid is too coarse to resolve the flow into this subrange and the simulation is in fact a "Very Large Eddy Simulation" (VLES). As the grid resolution increases, the part of the spectrum that follows the $-2 / 3$ law increases as more turbulence structures are directly resolved as expected in LES. Finally, for all grids, the spectra show the typical fast drop in energy as a result of the LES grid cut-off. A similar behaviour was noticed at different positions and heights and also in the no buildings case BL3-0. 


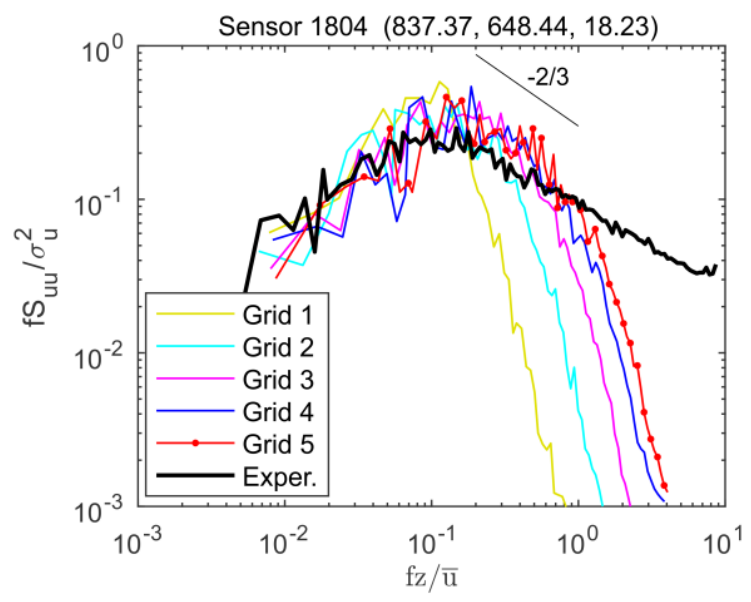

Fig. 7: Normalized energy density spectra of the streamwise velocity component at a single point, for all LES grids together with the spectrum derived from the experiment.

An estimation of the resolved amount of turbulence in an LES study can be made using the formula $k_{\text {res }} /\left(k_{\text {res }}+k_{s g s}\right)$ where $k_{\text {res }}$ is the resolved part of the turbulent kinetic energy and $k_{s g s}$ the subgrid part. This formula was applied in Grid 2 and Grid 5 case. The average value among all sensors is equal to 97.6 $\%$ and $99.2 \%$ for Grid 2 and Grid 5 respectively. In both cases the percentage of the resolved turbulent kinetic energy is very high. However, this alone is not representative of the quality of the LES simulation because neither the 'real' experimental turbulence nor the numerical diffusion, which in some cases can outweigh the SGS stresses, is considered.

\subsection{Detailed flow structure evaluation}

In this section, results from the simulation with Grid 5 are analysed and compared with the experiment in more detail. First, the exploratory data analysis of the preceding section is refined. Then, higher order statistics of the flow are evaluated alongside frequency distributions of the underlying instantaneous velocities.

\subsubsection{Statistical metrics and flow analysis}

Table 2 shows validation metrics for Grid 5 grouped based on different height levels in order to quantify in which regions of the domain the model performs best / worst. Statistical metrics are frequently used in order to quantify the quality of a model. However, the interpretation of their values needs to be made with caution and along with the overview of the flow field. For example, Koutsourakis (2014) reports misleading values at low heights and at some recirculation regions and Dejoan et al. (2010) suggest caution when interpreting statistical metrics since they can give similar values for different flow structures. Moreover, metrics values depend on the choice of the acceptance criteria, which can be application specific.

A moderate model performance is observed at the first two zones $(0-7 \mathrm{~m}$ and $7-14 \mathrm{~m})$. In these zones we expect the most complex flow phenomena. At higher levels metrics further improve. Acceptance criteria that are used by Hertwig et al. (2012) following the suggestions by VDI (2005) and Hanna et al. (2004) are FAC2 $\geq 0.5$ and $\mathrm{HR} \geq 0.66$. The threshold for FAC2 is satisfied in all zones for both velocity 
components whereas for HR it is met only sufficiently far away from the surface. The performance of the model is improved as the height increases according to both metrics.

In the work of Hertwig et al. (2012) the same experiment was simulated with RANS models using two different CFD codes. In that study, FAC2 estimated for all heights was equal to 0.78 for $\bar{u} / U_{\text {ref }}$ and 0.52 for $\bar{v} / U_{\text {ref }}$ (average values of the results for the two CFD codes). The values of the HR metric were equal to 0.59 and 0.6 , respectively. We observe that the LES of the present study overall performs better, which is particularly evident in the FAC2.

\begin{tabular}{|c|c|c|c|c|}
\hline & \multicolumn{2}{|c|}{$\overline{\bar{u}} / U_{r e f}$} & \multicolumn{2}{|c|}{$\bar{v} / U_{r e f}$} \\
\hline Height & FAC2 & HR & FAC2 & HR \\
\hline All heights & 0.84 & 0.55 & 0.77 & 0.69 \\
\hline $0-7 \mathrm{~m}$ & 0.69 & 0.30 & 0.64 & 0.39 \\
\hline $7-14 \mathrm{~m}$ & 0.68 & 0.46 & 0.75 & 0.62 \\
\hline $14-21 \mathrm{~m}$ & 0.90 & 0.33 & 0.84 & 0.77 \\
\hline $21-28 \mathrm{~m}$ & 0.99 & 0.75 & 0.86 & 0.88 \\
\hline $28-35 \mathrm{~m}$ & 1.00 & 0.88 & 0.86 & 0.88 \\
\hline$>35 \mathrm{~m}$ & 1.00 & 1.00 & 0.78 & 0.83 \\
\hline Ideal values & 1.00 & 1.00 & 1.00 & 1.00 \\
\hline
\end{tabular}

Table 2: Validation metrics at different height layer for the high-resolution Grid 5.

As indicated by the validation metrics, the most difficult area to predict the mean flow is inside the canopy layer, especially at low heights. In Fig. 8 the normalized mean velocity vectors at heights of 2 and $9 \mathrm{~m}$ are presented for both simulation and experiment. The prediction of the mean flow patterns is very satisfactory, especially concerning the flow direction. Regions of stronger wind speeds can be observed in the open area in lower left region of the domain. The flow exits the plaza mainly through a complex intersection on the right and it is subsequently channelled between buildings $\mathrm{O}$ and $\mathrm{L}$ and between buildings $\mathrm{H}$ and $\mathrm{L}$. A small part of the flow moves upwards between the buildings $\mathrm{B}$ and $\mathrm{G}$ and it meets the flow that comes from the upper side between the buildings $\mathrm{B}$ and $\mathrm{C}$.

At $2 \mathrm{~m}$ from the ground (Fig. 8, left), a strong flow reversal is formed between the buildings $\mathrm{G}$ and $\mathrm{H}$ due to the recirculation that occurs in street canyons with perpendicular wind forcing direction. A similar phenomenon is observed between buildings $\mathrm{L}$ and $\mathrm{M}$ as well as in the courtyards of buildings $\mathrm{G}$ and $\mathrm{D}$ due to a recirculation vortex forming there. Another interesting flow pattern is the anticlockwise horizontal vortex that is formed in the intersection area between buildings $\mathrm{H}$ and I. In all the above points, the simulation results are in overall very good agreement with the experiment. However, the underestimation of the velocity magnitude at several positions is evident too.

At $9 \mathrm{~m}$ from the ground (Fig. 8, right), the agreement with the experiment has improved, especially regarding the velocity magnitudes with noticeable exceptions in some areas such as between buildings $\mathrm{H}$ and $\mathrm{M}, \mathrm{H}$ and $\mathrm{E}$ and $\mathrm{M}$ and $\mathrm{I}$. In the areas where the velocity magnitudes are small, a strong $w$ velocity component is often expected due to the recirculation in the street canyons (the level of $9 \mathrm{~m}$ is about in the half of the average height of the buildings). Flow channelling along street canyons is established in several areas such as between buildings $\mathrm{C}$ and $\mathrm{G}$, and between $\mathrm{H}$ and $\mathrm{D}$. The flow between buildings $\mathrm{H}$ 
and D impacts on the downwind building E, which results in a splitting of the flow. Some stronger differences in wind speeds between the simulation and the experiment can be observed in this region of the domain (especially between buildings D and E) as a result of the complexity of the flow phenomena.
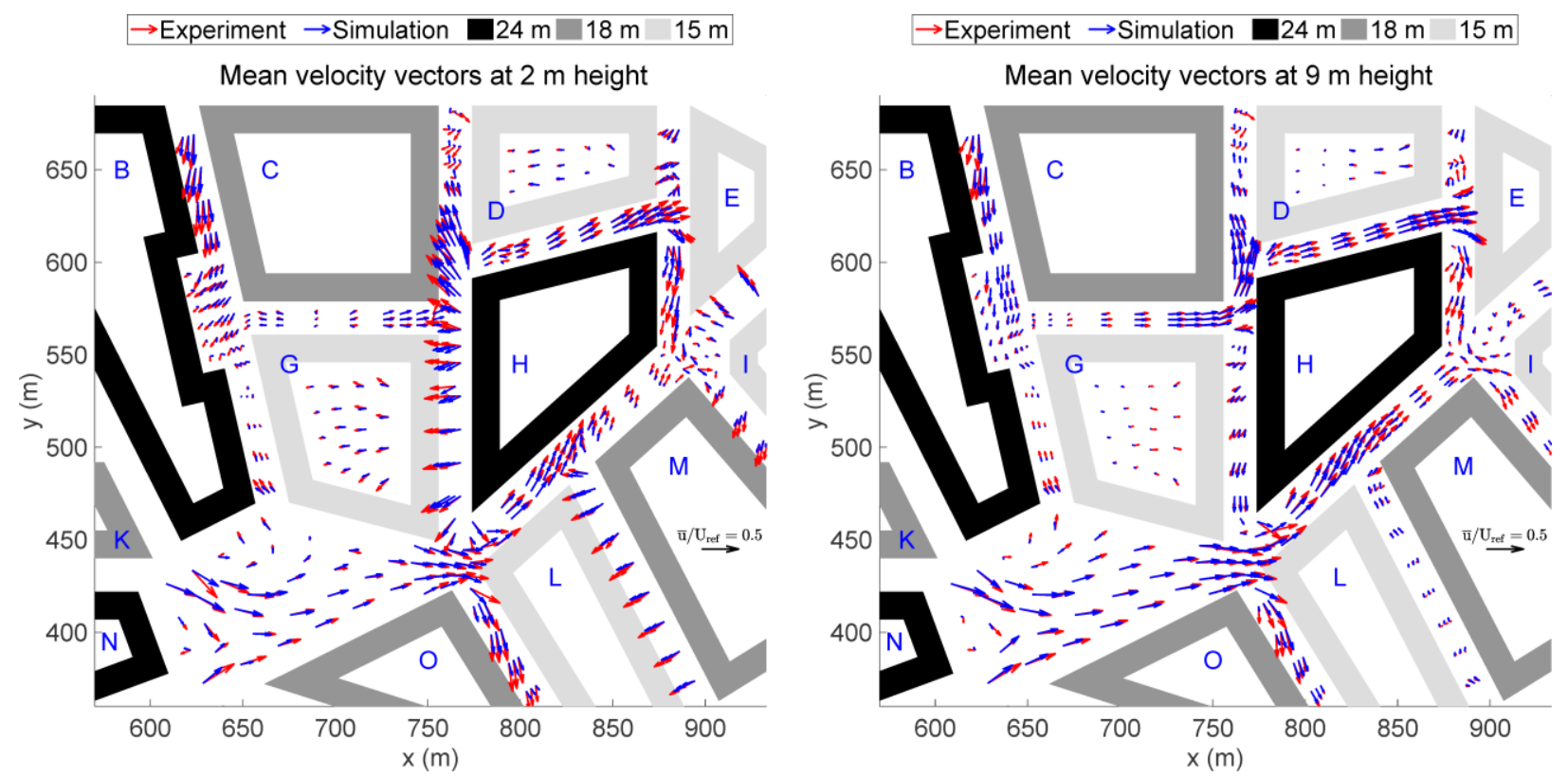

Fig. 8: Comparison between experimental and LES (Grid 5) results of the normalized mean velocity vectors at the sensor positions at 2 (left) and 9 (right) meters height. The approach flow is from left to right.

Observing the streamtraces of the mean flow can help to identify several flow features, both general and specific. In Fig. 9 overall ten different streamtraces are shown that provide information about the flow in the central part of the city. These were selected in order to illustrate the variety and complexity of flow behaviour in the model area. Horizontal velocity vectors in a plane at $\mathrm{z}=4.1 \mathrm{~m}$ are also included. Note that only every fourth vector is plotted in each direction for clarity. The streamtraces are coloured according to the local mean value of the streamwise velocity. Blue corresponds to backflow, while yellow/ red to positive velocities and thus in most cases also to higher altitudes. A general observation from this figure is that understanding the mean flow is not straightforward in such a complex geometry. Streamtraces starting from similar positions may proceed towards different directions (e.g streamtraces 1,2 and 6,7$)$ or follow complicated routes (e.g. 5, 8). Inside street canyons channelling effects can be observed and classical recirculation vortices depending on the orientation of the canyon with respect to the forcing wind direction. At oblique streets, the influence of both the channelling and the recirculation results in helical streamtraces (e.g. 6). 


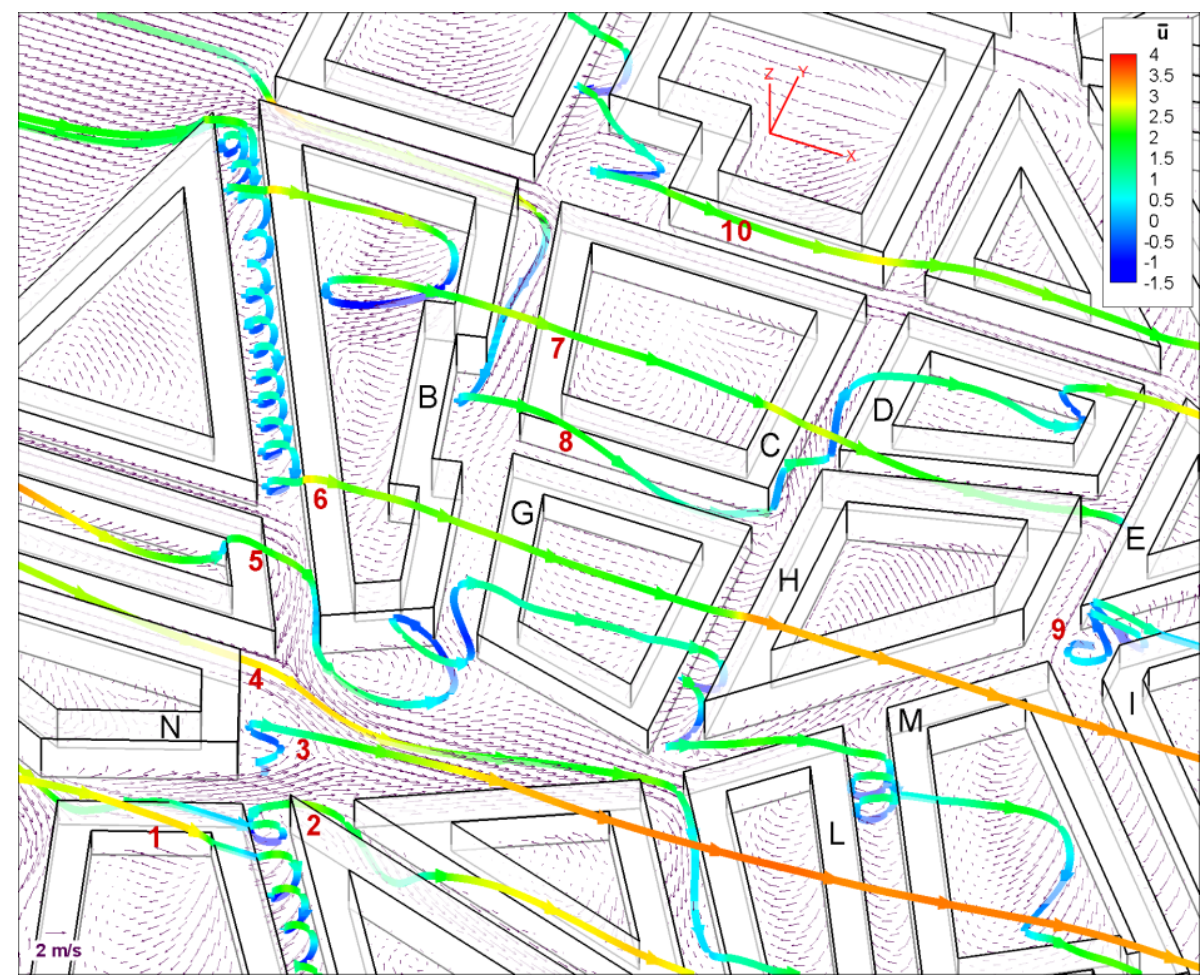

Fig. 9: LES (Grid 5) streamtraces of the mean flow at the centre of the model (colours indicate the local mean streamwise velocity), along with horizontal velocity vectors at $\mathrm{z}=4.1 \mathrm{~m}$ plane.

Streamtraces can also help to examine specific flow features. For example, behind building $\mathrm{N}$ a corner vortex with a vertical axis is formed (streamtrace 3) which disturbs the vorticity sheet shedding from the roof of building $\mathrm{N}$ (not seen here). A similar vertical vortex is formed at the intersection between buildings H, E, M, I (streamtrace 9). In this case, the flow is very complex and the position of the vortex is slightly oscillating. In the neighbourhood of the particular vortex, non-Gaussian velocity distributions can be found in both the experiment and the LES, as it will be shown later. This particular vertical vortex is gradually evolving into a horizontal one along the street canyon between buildings $\mathrm{E}$ and I. Something similar occurs in the vortex structure formed on the plaza below building B (streamtrace 5). In this example, the streamtrace completes half a loop between buildings B and G, then passes over the (low height) building $\mathrm{G}$, but does not manage to go over the taller building $\mathrm{H}$. Instead the flow is trapped at the canyon recirculation, then escapes above building $\mathrm{L}$ and loops again between buildings $\mathrm{L}$ and $\mathrm{M}$ and finally inside the courtyard of building $\mathrm{M}$, before escaping from the canopy layer.

Streamtrace 8 is another very illustrative example, since it passes through critical points of the central part of the city. Following this particular streamtrace, the flow enters the canyon between buildings B and C and then is lifted upwards at the notch of building B. In this particular region the flow is very complex due to influence of the flow from the neighbouring streets and to the complex building layout. NonGaussian velocity distributions can be found here. The streamtrace then goes over the recirculation vortex that is formed at the beginning of the street canyon between buildings $\mathrm{C}$ and $\mathrm{G}$, and goes down just after this vortex. Encountering building $\mathrm{H}$ the flow is split and the particular streamtrace goes upwards, at the street canyon between buildings $\mathrm{C}$ and $\mathrm{D}$, while also performing a couple of recirculations inside the canyon and later in the courtyard of building $\mathrm{D}$. This is a particularly good example for the strong three- 
dimensional nature of the urban flow field, in which the flow (and e.g. pollutants carried with the flow) can leave and re-enter the canopy layer, resulting in significant vertical interaction.

Another example of trapping in courtyards, which are a prominent feature of the "Michel-Stadt" geometry and characteristic for many European cities, is streamtrace 7 (courtyard of building B). Other streamtraces, not shown here, reveal that the flow can be trapped inside such yards for a very long time. After streamtrace 7 has exited the courtyard, it moves in a high elevation before diving into the canyon between buildings $\mathrm{D}$ and $\mathrm{H}$.

\subsubsection{Second and third order statistics and velocity distributions}

In Fig. 10, the scatter plots for the normalized second order statistics of the velocity components are presented for the layer in which turbulence has developed mainly due to the presence of the buildings (height below $28 \mathrm{~m}$ ). The results have been grouped according to selected height ranges and concern the resolved part of turbulence. The SGS part of turbulence is neglected, since it is on average less than 1\%, as commented at the end of section 4.1., From Fig. 10, we observe that in general the turbulent stresses are under-predicted. At elevations higher than $28 \mathrm{~m}$, the under-prediction is larger.
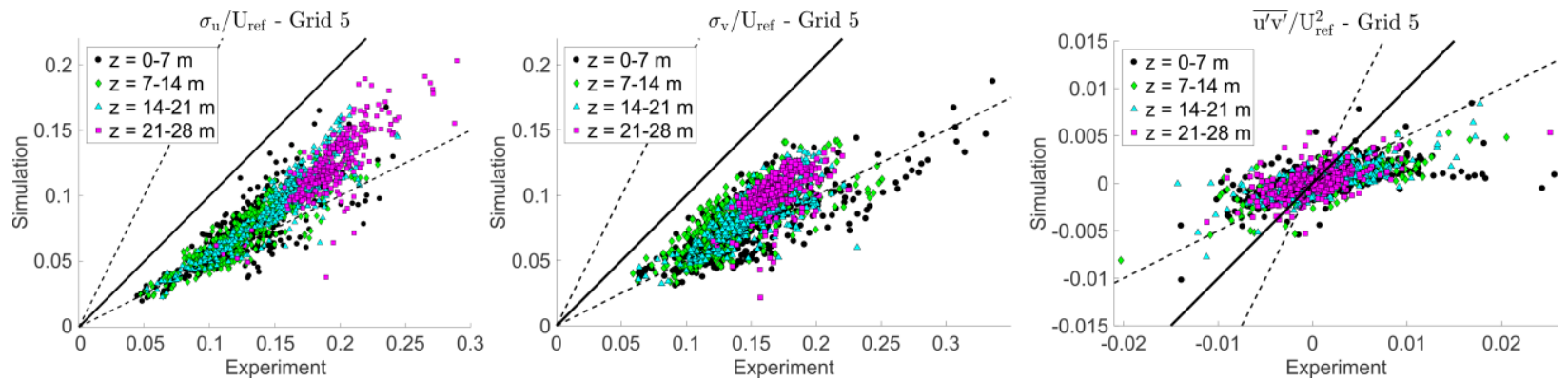

Fig. 10: Scatter plots for the normalized standard deviation of the $u$ and $v$ velocity components and the $\overline{u^{\prime} v^{\prime}}$ stresses for Grid 5. The dashed lines indicate the 1-to-2 and 2-to-1 margins.

In order to evaluate some possible reasons for the divergence of the predicted stresses from the measurements, various simulation sensitivity analyses were performed. For this analysis the resolution of the coarser Grid 3 was used to save computational time. The first possible reason that was examined was the inlet turbulence. In particular, the effect of the standard deviation required by the Langevin-type boundary condition was tested. Doubling the values of the standard deviation did not reveal any significant impact on the mean velocity profiles. On the other hand small differences were observed for the Reynold stresses $\overline{u^{\prime} u^{\prime}}$ where higher values were predicted especially at high elevations. However these values were still much lower than the experimental ones. At lower heights $(<20 \mathrm{~m})$ the differences were small at the majority of the comparison sites. This behaviour was expected because at greater heights the results are more strongly affected by the imposed fluctuations at the top and inlet boundaries, whereas inside the city turbulence is self-developed. The Reynolds stresses $\overline{v^{\prime} v^{\prime}}$ seemed to remain almost unaffected at higher elevations whereas small differences were observed at lower heights. The sensitivity of the results on the autocorrelation time scale parameter of the Langevin-type boundary condition was also investigated. When using a five times smaller time scale, the observed effect on the results was smaller than the effect of the higher standard deviation.

Another sensitivity test had to do with the value of Smagorinsky constant $C_{s}$. The specific test was conducted with Grid 2, because the influence of $C_{s}$ is more pronounced at coarser grids. Using $C_{s}=0.2$ 
instead of 0.1 , resulted in a significant increase of the subgrid-scale turbulence values (approximately 3.5 times higher SGS turbulent velocity). On the other hand, the resolved part of turbulence was severely damped in most areas of the domain. Thus, the overall turbulence was on average lower and the agreement with the experiment was worse: FAC2 for $\sigma_{u}$ dropped from 0.46 to 0.38 and for $\bar{u}$ from 0.70 to 0.65 . Thus $C_{s}=0.1$, which is the most common value used in the literature, provided better results in this study too.

Another possible reason for the disagreement with the experiment regarding the Reynolds stresses is the domain length. If the inlet boundary is too close to the area of interest, turbulence (especially far from solids) may not have the appropriate length to fully develop, even if an artificial turbulence generation method is used at the inlet. In order to examine the sensitivity of the results on the inlet boundary position, the boundary was moved $1500 \mathrm{~m}$ upwind of the city (using Grid 3 resolution). Furthermore, in the area in front of the city, a series of roughness elements ( 24 columns of obstacles that also existed in the wind tunnel experiment) were positioned, in order to assist in the turbulence generation. In this case, there was a small enhancement of the stresses at medium and large heights of the domain. However, the values at the sensors positions, especially inside the city, did not have significant increase and were still low compared to the experiment.

Finally, different numerical schemes were tested using Grid 3, like Cubic Upwind, FROMM, QUICK, MUSCL, SMART, STOIC and van Albada (Waterson and Deconinck 2007), even if not all of them are actually popular for LES. The cubic upwind and the FROMM schemes achieved the best agreement (in terms of $\mathrm{HR}$ and FAC2 metrics) with the experiment for both mean velocity field and stresses. The differences regarding the mean velocities were small but the differences regarding the stresses were more significant. The FAC2 metric for all sensors for the standard deviation of $u$ in the bounded central differences case (initial scheme, Grid 3) is equal to 0.60 whereas in the FROMM case 0.73. The differences in the standard deviation of $v$ are bigger; FAC2 increases from 0.55 to 0.72 . Even though the predictions are still not in very good agreement with the experiment, it is obvious that the choice of the numerical scheme is very significant. A less diffusive scheme might have produced better results. The central differences scheme, which is quite popular in LES, could not be used due to the complex geometry (convergence issues). In the case without buildings (BL3-0) where the central differences scheme was used, turbulent stresses agreed much better with the experiment.

In Fig. 11 contours of the third-order moment (skewness) of the $u$ and $v$ velocity components are presented for the simulation and the experiment at $2 \mathrm{~m}$ height. The contours have been estimated based on the values at the sensor positions (see Fig. 2, left) for both experiment and simulation. The skewness describes the shape of the distribution curve and is a measure of asymmetry of the distribution compared to the symmetric case of a normal (Gaussian) reference. Large absolute values of skewness indicate that long tails exists, i.e. the distribution is skewed towards low or high values. This parameter, hence, is an important indicator of infrequently occurring very high (or very low) velocities at a certain location and thus of flow unsteadiness.

In the figure, we observe that except for some areas close to building corners and some street canyons, there are noticeable differences between the LES and the experiment. This is something that we should expect due to the under-prediction of the second-order statistics. However, some striking similarities are also observed. Regions with non-zero skewness can be identified in both simulation and experiment. For example positive skewness in the $u$ velocity component is observed between the buildings $\mathrm{H}$ and $\mathrm{D}$ (tail 
towards high velocities) and negative between $\mathrm{H}$ and $\mathrm{E}$ (tail towards low velocities). In the $v$ velocity component, a pair of negative and positive skewness regions is formed between the buildings $\mathrm{D}$ and $\mathrm{E}$. At higher elevations (9 and $18 \mathrm{~m}$, see supplementary materials) we find the agreement of LES results with the experimental data to noticeably increase compared to the $2 \mathrm{~m}$ layer.

In order to understand the shape of the underlying distributions better, Fig. 12 shows histograms of the instantaneous horizontal velocity components at three locations that are indicated by circles in Fig. 11. We observe that LES is not only capable in predicting general non-Gaussian distributions but also bimodal distributions. In Point 1 we can see that the simulation predicts a bimodal-like distribution of the $u$ velocity, which is similar to that of the experiment. At Point 2 a negatively skewed distribution is found for the $v$ component, which agrees very well with the experiment. The simulation represents also very well the bimodal distribution of the $v$ component at Point 3 found in the experiment. Bimodal distributions appear also at the neighbouring points (not shown). This indicates that the flow in this area is highly unsteady. An interesting remark is that bimodal distributions usually occur between areas of high negative and high positive skewness (Points 1, 3; Fig. 11 and Fig. 12). In the case of Point 1, the bimodal distribution appears in the region where an unstable vortex is formed (see also Fig. 8 and Fig. 9). The complex layout of the area (intersection of four streets) contributes to the formation of this unsteady vortex.

It is worth to note that the bimodal distributions predicted in Point 3 and in two neighbouring points, are not predicted by the coarser computational grids. The same is true for Point 1 - the simulation with Grid 3 however, predicts two bimodal-like distributions (see supplementary materials) near that point. In other positions, fewer and less striking bimodal distributions are predicted with the coarser grids, emerging in areas where such distributions are not found in the experiment (only one bimodal distribution is predicted near an experimental one at the beginning of the street canyon between buildings L and $\mathrm{H}$, using grid 4). Consequently, it generally seems that the denser the grid, the higher its ability to predict more satisfactorily the occurrence of unsteady flow switching phenomena.

Bimodal distribution means that the flow switches between two mean states. This is a characteristic of the unsteadiness of the flow. Steady-state RANS models cannot predict the effects of such phenomena. The capability of LES to predict the flow unsteadiness is one of its great advantages over RANS. A significant remark that needs to be made is that the existence of bimodal distributions at several points of the flow field means that the "mean" flow may locally not have a physical meaning, because the mean value is not representative of either peak in the distribution. The same is true for higher order statistics. This was also highlighted by Hertwig et al. (2012). In this context, streamtraces like those presented in Fig. 9 should be carefully interpreted in case they pass through highly unstable regions. 

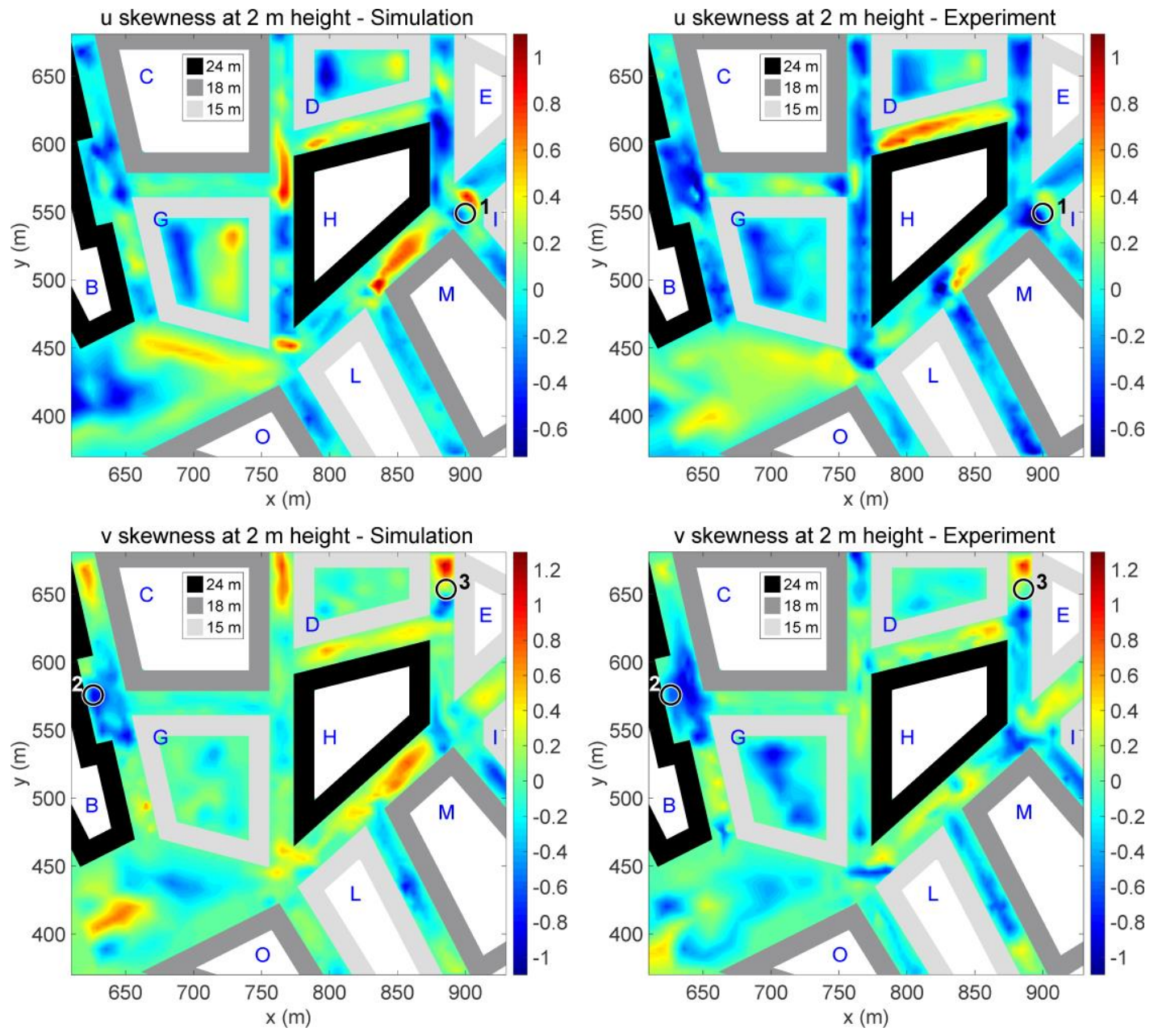

Fig. 11: LES (left; Grid 5 results) and experimental (right) skewness contours of the u (top) and v (bottom) velocity components at $2 \mathrm{~m}$ height. 

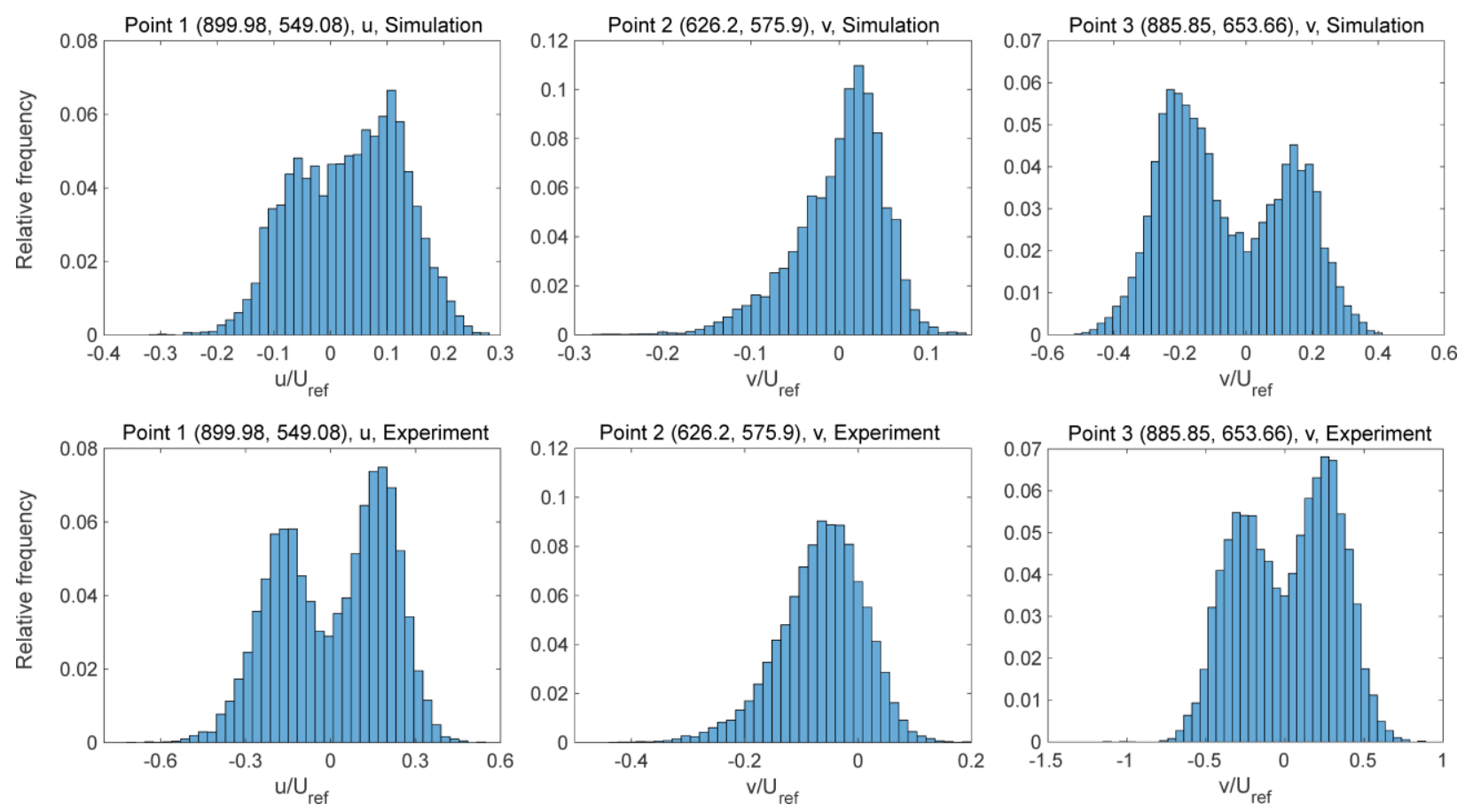

Fig. 12: Frequency distribution of horizontal velocity components for LES (top; Grid 5) and experiment (bottom) at the highlighted locations shown in Fig. 11.

\section{Conclusions}

The CEDVAL-LES wind tunnel database was used for the evaluation of the ADREA-HF LES code. The particular database includes cases that are suitable for both urban LES code validation and the study of complex-geometry flow features. In this study, one dataset was tested, the "Michel-Stadt" semi-idealized city, which provides a unique opportunity to study an almost real city geometry, without the oversimplifications of regular arrays of obstacles often used as city-models. The LES study of the flow in a whole city is a challenge due to the complexity of the geometry and the high demands in computational power. In this work, the LES method used was found to be suitable for the study of turbulent flow in the urban environment and valuable conclusions were deduced.

An extensive grid sensitivity study was performed in order to assess the grid impact on the results. Obviously, higher resolutions reveal more detailed flow structures (and evident bimodal velocity distributions) and take into account the building details with higher accuracy. The study showed that with regard to general flow features a resolution around $1.5 \mathrm{~m}$ in this test case produced results that are fairly "grid-independent".

The Langevin-type boundary condition was used at the inflow plane in order to produce inlet turbulence for LES. This method has significant advantages over the cyclic boundary condition and it was used with success. An originality of this study is the use of turbulent fluctuations at the top of the domain (again using the Langevin-type boundary condition), thus avoiding the turbulence damping that other top boundary conditions may have and being able to reduce the number of vertical cells. 
The agreement with the experiment was generally good and this encourages the use of the particular code for more urban LES studies. The mean velocities overall were predicted accurately, as it was shown from the vertical profiles, scatter plots and velocity vectors. This was also shown through the statistical performance measures that were used to quantify the agreement. However, an underestimation of the streamwise velocity was observed at several sites in the city. The LES also underestimated more severely the Reynolds stresses: the numerical scheme that was used is believed to have strongly contributed to this result.

The study of streamtraces offered a practical way to examine the general flow over the central part of the city. It also revealed the complex nature of the flow in cities and helped to identify many specific features like helical vortices, flow splitting, vertical-axis vortices, flow leaving and re-entering the canopy layer, etc. Diagrams like those give a general overview of the flow and should be included in every CFD validation because the evaluation of a code based only in statistical metrics can lead to misleading conclusions. More streamtraces, not presented here, revealed additional interesting flow characteristics like co-axial helical vortices moving towards opposite directions.

A significant finding of this work is the fact that LES is capable to accurately predict non-Gaussian velocity distributions and more importantly bimodal distributions, which are typical for urban flow behaviour, for example in intersections. This is a major strength of LES compared to steady-state RANS methods. Areas that are likely to feature bimodal distributions of velocities are those where the flow is highly unsteady. In this study, skewness contours were also compared with the experiment. It is shown that areas where local regions of negative and positive skewness of the velocity exist are likely to exhibit bimodal distribution. In those areas, and generally in regions of high absolute skewness and non-Gaussian velocity distributions, the "mean" flow does not actually have a physical meaning and thus the use of RANS is inappropriate.

As future work, even more extensive LES validation and analysis of turbulent flow, like for example that suggested by Hertwig et al. (2017a, b) could be done. This approach gives emphasis on the timedependent characteristics of the flow. Also more geometry-induced flow features like coherent structures that are formed within and above the city could be studied. In general, LES has a strong potential to significantly advance our current understanding of urban aerodynamics particularly regarding the nature and impact of unsteady flows.

\section{Acknowledgments}

This work was initially supported from IKYDA (Programme for the promotion of the exchange and scientific cooperation between Greece and Germany), in the framework of the "MODEX" (Modelling individual exposure from airborne hazardous releases) project. The authors would like to thank prof. Bernd Leitl at the University of Hamburg for his assistance with the experimental data. This work was also supported by computational time granted from the Greek Research \& Technology Network (GRNET) in the Greek National HPC facility ARIS (http://hpc.grnet.gr) under projects FLUEN (pr001016) and CFD-URB (pr004009). NK would also like to thank the Greek Scholarships' Foundation 
and the Municipality of Aghia Varvara Attikis, Greece, for their financial support and prof. Nicolas C. Markatos for his advice during the beginning of this study.

\section{References}

Argyropoulos, C.D., Markatos, N.C., 2015. Recent advances on the numerical modelling of turbulent flows. Appl. Math. Model. https://doi.org/10.1016/j.apm.2014.07.001

Aristodemou, E., Boganegra, L.M., Mottet, L., Pavlidis, D., Constantinou, A., Pain, C., Robins, A., ApSimon, H., 2018. How tall buildings affect turbulent air flows and dispersion of pollution within a neighbourhood. Environ. Pollut. 233, 782-796. https://doi.org/10.1016/J.ENVPOL.2017.10.041

Bartzis, J., 1991. ADREA-HF: a three-dimensional finite volume code for vapour cloud dispersion in complex terrain. EUR Rep. 13580 EN.

Bastigkeit, I., 2011. Erzeugung von Validierungsdaten fur wirbelauflosende mikroskalige Stromungs-und Ausbreitungsmodelle. PhD dissertation, Department Geowissenschaften, Universitat Hamburg.

Blocken, B., 2015. Computational Fluid Dynamics for urban physics: Importance, scales, possibilities, limitations and ten tips and tricks towards accurate and reliable simulations. Build. Environ. 91, 219-245. https://doi.org/10.1016/J.BUILDENV.2015.02.015

Boppana, V.B.L., Xie, Z.T., Castro, I.P., 2010. Large-eddy simulation of dispersion from surface sources in arrays of obstacles. Boundary-Layer Meteorol. 135, 433-454. https://doi.org/10.1007/s10546-010-9489-9

Bou-Zeid, E., Overney, J., Rogers, B.D., Parlange, M.B., 2009. The Effects of Building Representation and Clustering in Large-Eddy Simulations of Flows in Urban Canopies. Boundary-Layer Meteorol. 132, 415-436. https://doi.org/10.1007/s10546-009-9410-6

Camelli, F.., Lohner, R., Hanna, S.R., 2006. VLES Study of Flow and Dispersion Patterns in Heterogeneous Urban Areas, in: AIAA Paper 2006-1419, 44th AIAA Aerospace Sciences Meeting and Exhibit, 9 - 12 January. Reno, Nevada.

Castro, I.P., Xie, Z.-T., Fuka, V., Robins, A.G., Carpentieri, M., Hayden, P., Hertwig, D., Coceal, O., 2017. Measurements and Computations of Flow in an Urban Street System. Boundary-Layer Meteorol. 162, 207230. https://doi.org/10.1007/s10546-016-0200-7

Davidson, L., 2005. Hybrid LES-RANS: inlet boundary conditions, in: Skallerud, B., Andersson, H.I. (Eds.), Proceedings of the 3rd National Conference on Computational Mechanics, MekIT05, 11-22 May. Trondheim, Norway, pp. 7-22.

Dejoan, A., Santiago, J.L., Martilli, A., Martin, F., Pinelli, A., 2010. Comparison Between Large-Eddy Simulation and Reynolds-Averaged Navier-Stokes Computations for the MUST Field Experiment. Part II: Effects of Incident Wind Angle Deviation on the Mean Flow and Plume Dispersion. Boundary-Layer Meteorol. 135, 133-150. https://doi.org/10.1007/s10546-010-9467-2

Efthimiou, G.C., Andronopoulos, S., Bartzis, J.G., 2017a. Prediction of dosage-based parameters from the puff dispersion of airborne materials in urban environments using the CFD-RANS methodology. Meteorol. Atmos. Phys. 1-18. https://doi.org/10.1007/s00703-017-0506-0

Efthimiou, G.C., Andronopoulos, S., Bartzis, J.G., Berbekar, E., Harms, F., Leitl, B., 2017b. CFD-RANS prediction of individual exposure from continuous release of hazardous airborne materials in complex urban environments. J. Turbul. 18, 115-137. https://doi.org/10.1080/14685248.2016.1246736

Efthimiou, G.C., Hertwig, D., Andronopoulos, S., Bartzis, J.G., Coceal, O., 2017c. A Statistical Model for the Prediction of Wind-Speed Probabilities in the Atmospheric Surface Layer. Boundary-Layer Meteorol. 163, 179-201. https://doi.org/10.1007/s10546-016-0221-2

Elshaer, A., Aboshosha, H., Bitsuamlak, G., El Damatty, A., Dagnew, A., 2016. LES evaluation of wind-induced 
responses for an isolated and a surrounded tall building. Eng. Struct. 115, 179-195. https://doi.org/10.1016/J.ENGSTRUCT.2016.02.026

Erlebacher, G., Hussaini, M.Y., Speziale, C.G., Zang, T.A., 1992. Toward the large-eddy simulation of compressible turbulent flows. J. Fluid Mech. 238, 155-185. https://doi.org/10.1017/S0022112092001678

Fischer, R., Bastigkeit, I., Leitl, B., Schatzmann, M., 2010. Generation of spatio-temporally high resolved datasets for the validation of LES-models simulating flow and dispersion phenomena within the lower atmospheric boundary layer, in: Proceedings of the Fifth International Symposium on Computational Wind Engineering (CWE2010) Chapel Hill, North Carolina, USA May 23-27.

Fuka, V., Brechler, J., 2014. Large Eddy Simulation of Accidental Releases of Hazardous Substances in Idealized Urban Geometry, in: Batchvarova, E., Kirova, H., and Hristova, E., Editors, 16th Conference on Harmonisation within Atmospheric Dispersion Modelling for Regulatory Purposes. National Institute of Meteorology and Hydrology Bulgarian Academy of Sciences, September 2014. pp. 709-711.

Fuka, V., Xie, Z.-T., Castro, I.P., Hayden, P., Carpentieri, M., Robins, A.G., 2018. Scalar Fluxes Near a Tall Building in an Aligned Array of Rectangular Buildings. Boundary-Layer Meteorol. 167, 53-76. https://doi.org/10.1007/s10546-017-0308-4

Gariazzo, C., Leitl, B., Trini Castelli, S., Baumann-Stanzer, K., Reisin, T.G., Barmpas, F., Tinarelli, G., Milliez, C.M., Armand, P., Bemporad, E., 2014. Cost action ES1006. Evaluation, improvement and guidance of localscale emergency prediction and response tools for airborne hazards in built environments: ongoing activities, experiments and recent results. Chem. Eng. Trans. 36, 529-534. https://doi.org/10.3303/CET1436089

Giometto, M.G., Christen, A., Meneveau, C., Fang, J., Krafczyk, M., Parlange, M.B., 2016. Spatial Characteristics of Roughness Sublayer Mean Flow and Turbulence Over a Realistic Urban Surface. Boundary-Layer Meteorol. 160, 425-452. https://doi.org/10.1007/s10546-016-0157-6

Goodfriend, E., Katopodes Chow, F., Vanella, M., Balaras, E., 2016. Large-Eddy Simulation of Flow Through an Array of Cubes with Local Grid Refinement. Boundary-Layer Meteorol. 159, 285-303. https://doi.org/10.1007/s10546-016-0128-y

Gousseau, P., Blocken, B., Stathopoulos, T., van Heijst, G.J.F., 2015. Near-field pollutant dispersion in an actual urban area: Analysis of the mass transport mechanism by high-resolution Large Eddy Simulations. Comput. Fluids 114, 151-162. https://doi.org/10.1016/J.COMPFLUID.2015.02.018

Gousseau, P., Blocken, B., Stathopoulos, T., van Heijst, G.J.F., 2011. CFD simulation of near-field pollutant dispersion on a high-resolution grid: A case study by LES and RANS for a building group in downtown Montreal. Atmos. Environ. 45, 428-438. https://doi.org/10.1016/J.ATMOSENV.2010.09.065

Gu, Z.L., Jiao, J.Y., Su, J.W., 2011. Large-Eddy Simulation of the Wind Field and Plume Dispersion Within Different Obstacle Arrays Using a Dynamic Mixing Length Subgrid-Scale Model. Boundary-Layer Meteorol. 139, 439-455. https://doi.org/10.1007/s10546-010-9587-8

Hanna, S.R., Hansen, O.R., Dharmavaram, S., 2004. FLACS CFD air quality model performance evaluation with Kit Fox, MUST, Prairie Grass, and EMU observations. Atmos. Environ. 38, 4675-4687. https://doi.org/10.1016/j.atmosenv.2004.05.041

Hanna, S.R., Tehranian, S., Carissimo, B., Macdonald, R., Lohner, R., 2002. Comparisons of model simulations with observations of mean flow and turbulence within simple obstacle arrays. Atmos. Environ. 36, 50675079. https://doi.org/10.1016/S1352-2310(02)00566-6

He, J., Song, C.C.., 1999. Evaluation of pedestrian winds in urban area by numerical approach. J. Wind Eng. Ind. Aerodyn. 81, 295-309. https://doi.org/10.1016/S0167-6105(99)00025-2

Hertwig, D., Efthimiou, G.C., Bartzis, J.G., Leitl, B., 2012. CFD-RANS model validation of turbulent flow in a semi-idealized urban canopy. J. Wind Eng. Ind. Aerodyn. 111, 61-72. https://doi.org/10.1016/j.jweia.2012.09.003

Hertwig, D., Leitl, B., Schatzmann, M., 2011. Organized turbulent structures-Link between experimental data and LES. J. Wind Eng. Ind. Aerodyn. 99, 296-307. https://doi.org/10.1016/j.jweia.2011.01.002

Hertwig, D., Patnaik, G., Leitl, B., 2017a. LES validation of urban flow, part I: flow statistics and frequency 
distributions. Environ. Fluid Mech. 17, 521-550. https://doi.org/10.1007/s10652-016-9507-7

Hertwig, D., Patnaik, G., Leitl, B., 2017b. LES validation of urban flow, part II: eddy statistics and flow structures. Environ. Fluid Mech. 17, 551-578. https://doi.org/10.1007/s10652-016-9504-x

Ikegaya, N., Ikeda, Y., Hagishima, A., Tanimoto, J., 2017. Evaluation of rare velocity at a pedestrian level due to turbulence in a neutrally stable shear flow over simplified urban arrays. J. Wind Eng. Ind. Aerodyn. 171, 137147. https://doi.org/10.1016/J.JWEIA.2017.10.002

Inagaki, A., Castillo, M.C.L., Yamashita, Y., Kanda, M., Takimoto, H., 2012. Large-Eddy Simulation of Coherent Flow Structures within a Cubical Canopy. Boundary-Layer Meteorol. 142, 207-222. https://doi.org/10.1007/s10546-011-9671-8

Jarrin, N., Benhamadouche, S., Laurence, D., Prosser, R., 2006. A synthetic-eddy-method for generating inflow conditions for large-eddy simulations. Int. J. Heat Fluid Flow 27, 585-593. https://doi.org/10.1016/j.ijheatfluidflow.2006.02.006

Kanda, M., Inagaki, A., Miyamoto, T., Gryschka, M., Raasch, S., 2013. A New Aerodynamic Parametrization for Real Urban Surfaces. Boundary-Layer Meteorol. 148, 357-377. https://doi.org/10.1007/s10546-013-9818-x

Klein, M., Sadiki, A., Janicka, J., 2003. A digital filter based generation of inflow data for spatially developing direct numerical or large eddy simulations. J. Comput. Phys. 186, 652-665. https://doi.org/10.1016/S00219991(03)00090-1

Koutsourakis, N., 2014. Turbulent flow and pollutant dispersion in the urban environment. PhD thesis (in Greek), Department of Mechanical Engineering, University of Western Macedonia, Kozani, Greece, https://www.didaktorika.gr/eadd/handle/10442/35304.

Koutsourakis, N., Hertwig, D., Efthimiou, G.C., Venetsanos, A.G., Bartzis, J.G., Leitl, B., 2012. Evaluation of the ADREA-HF LES code for urban air quality assessment, using the CEDVAL-LES wind tunnel database, in: Proc. 8th Int. Conf. on Air Quality - Science and Application. Athens, 19-23 March.

Kovalets, I. V., Andronopoulos, S., Venetsanos, A.G., Bartzis, J.G., 2008. Optimization of the numerical algorithms of the ADREA-I mesoscale prognostic meteorological model for real-time applications. Environ. Model. Softw. 23, 96-108. https://doi.org/10.1016/j.envsoft.2007.05.004

Leitl, B., Harms, F., Berbekar, E., Boris, J., Patnaik, G., Obenschain, K., Fischer, S., 2016. Local-scale hazmat dispersion modelling for first responders based on high-resolution computational fluid dynamics - an overview of CT-Analyst Hamburg. Chem. Eng. Trans. 48, 43-48. https://doi.org/10.3303/CET1648008

Leitl, B., Hertwig, D., Harms, F., Schatzmann, M., Patnaik, G., Boris, J., Obenschain, K., Fischer, S., Rechenbach, P., 2014. Large eddy simulation of accidental releases, in: Talamelli A., Oberlack M., Peinke J. (Eds) Progress in Turbulence V. Springer Proceedings in Physics. Springer, Cham, pp. 133-147. https://doi.org/10.1007/9783-319-01860-7_22

Letzel, M.O., Helmke, C., Ng, E., An, X., Lai, A., Raasch, S., 2012. LES case study on pedestrian level ventilation in two neighbourhoods in Hong Kong. Meteorol. Zeitschrift 21, 575-589.

Liu, Y.S., Cui, G.X., Wang, Z.S., Zhang, Z.S., 2011. Large eddy simulation of wind field and pollutant dispersion in downtown Macao. Atmos. Environ. 45, 2849-2859. https://doi.org/10.1016/J.ATMOSENV.2011.03.001

Liu, Y.S., Miao, S.G., Zhang, C.L., Cui, G.X., Zhang, Z.S., 2012. Study on micro-atmospheric environment by coupling large eddy simulation with mesoscale model. J. Wind Eng. Ind. Aerodyn. 107-108, 106-117. https://doi.org/10.1016/J.JWEIA.2012.03.033

Llaguno-Munitxa, M., Bou-Zeid, E., Hultmark, M., 2017. The influence of building geometry on street canyon air flow: Validation of large eddy simulations against wind tunnel experiments. J. Wind Eng. Ind. Aerodyn. 165, 115-130. https://doi.org/10.1016/J.JWEIA.2017.03.007

Lund, T.S., Wu, X., Squires, K.D., 1998. Generation of Turbulent Inflow Data for Spatially-Developing Boundary Layer Simulations. J. Comput. Phys. 140, 233-258. https://doi.org/10.1006/jcph.1998.5882

Mathey, F., Cokljat, D., Bertoglio, J.P., Sergent, E., 2006. Assessment of the vortex method for Large Eddy Simulation inlet conditions. Prog. Comput. Fluid Dyn. An Int. J. 6, 58-67. 
https://doi.org/10.1504/PCFD.2006.009483

Michioka, T., Sato, A., Sada, K., 2013. Large-eddy simulation coupled to mesoscale meteorological model for gas dispersion in an urban district. Atmos. Environ. 75, 153-162. https://doi.org/10.1016/J.ATMOSENV.2013.04.017

Michioka, T., Takimoto, H., Sato, A., 2014. Large-Eddy Simulation of Pollutant Removal from a ThreeDimensional Street Canyon. Boundary-Layer Meteorol. 150, 259-275. https://doi.org/10.1007/s10546-0139870-6

Moon, K., Hwang, J.-M., Kim, B.-G., Lee, C., Choi, J., 2014. Large-eddy simulation of turbulent flow and dispersion over a complex urban street canyon. Environ. Fluid Mech. 14, 1381-1403. https://doi.org/10.1007/s10652-013-9331-2

Murakami, S., 1998. Overview of turbulence models applied in CWE-1997. J. Wind Eng. Ind. Aerodyn. 74-76, 124. https://doi.org/10.1016/S0167-6105(98)00004-X

Nakayama, H., Leitl, B., Harms, F., Nagai, H., 2014. Development of local-scale high-resolution atmospheric dispersion model using large-eddy simulation. Part 4: turbulent flows and plume dispersion in an actual urban area. J. Nucl. Sci. Technol. 51, 626-638. https://doi.org/10.1080/00223131.2014.885400

Nakayama, H., Nagai, H., 2011. Large-Eddy Simulation of Plume Dispersion within a Regular Array of Cubic Buildings. Prog. Nucl. Sci. Technol. 2, 463-469.

Nakayama, H., Takemi, T., Nagai, H., 2012. Large-eddy simulation of urban boundary-layer flows by generating turbulent inflows from mesoscale meteorological simulations. Atmos. Sci. Lett. 13, 180-186. https://doi.org/10.1002/asl.377

Nozawa, K., Tamura, T., 2002. Large eddy simulation of the flow around a low-rise building immersed in a roughwall turbulent boundary layer. J. Wind Eng. Ind. Aerodyn. 90, 1151-1162. https://doi.org/10.1016/S01676105(02)00228-3

Nozu, T., Tamura, T., 2012. LES of turbulent wind and gas dispersion in a city. J. Wind Eng. Ind. Aerodyn. 104106, 492-499. https://doi.org/10.1016/J.JWEIA.2012.02.024

Nozu, T., Tamura, T., Okuda, Y., Sanada, S., 2008. LES of the flow and building wall pressures in the center of Tokyo. J. Wind Eng. Ind. Aerodyn. 96, 1762-1773. https://doi.org/10.1016/J.JWEIA.2008.02.028

Nozu, T., Tamura, T., Takeshi, K., Akira, K., 2015. Mesh-adaptive LES for wind load estimation of a high-rise building in a city. J. Wind Eng. Ind. Aerodyn. 144, 62-69. https://doi.org/10.1016/j.jweia.2015.05.007

Park, S.-B., Baik, J.-J., Han, B.-S., 2015. Large-eddy simulation of turbulent flow in a densely built-up urban area. Environ. Fluid Mech. 15, 235-250. https://doi.org/10.1007/s10652-013-9306-3

Patnaik, G., Boris, J.P., Grinstein, F.F., Iselin, J.P., Hertwig, D., 2012. Large Scale Urban Simulations with FCT. Springer, Dordrecht, pp. 91-117. https://doi.org/10.1007/978-94-007-4038-9_4

Piomelli, U., 2001. Large-eddy and direct simulation of turbulent flows. Short course delivered at CFD2001 - 9e conference annuelle de la Societe canadienne de CFD, May 2001, Kitchener, Ontario, Available online from http://terpconnect.umd.edu/ ugo/research/publications.html.

Saad, Y., 2003. Iterative methods for sparse linear systems, 2nd ed. Society for Industrial and Applied Mathematics, http://www-users.cs.umn.edu/ saad/IterMethBook_2ndEd.pdf.

Saeedi, M., Wang, B.-C., 2015. Large-eddy simulation of turbulent flow and dispersion over a matrix of wallmounted cubes. Phys. Fluids 27, 115104. https://doi.org/10.1063/1.4935112

Sagaut, P., 2006. Large eddy simulation for incompressible flows: An introduction, 3rd ed.

Schatzmann, M., Olesen, H., Franke, J., 2010. Model evaluation case studies: approach and results. COST Action 732 Quality assurance and improvement of microscale meteorological models, ISBN: 3-00-018312-4. University of Hamburg.

Sergent, M.E., 2002. Vers une Methodologie de Couplage Entre la Simulation des Grandes Echelles et les Modeles Statistiques. Phd thesis, Ecole Central de Lyon, Lyon, France. 
Smagorinsky, J., 1963. General circulation experiments with the primitive equations. I. The basic experiment. Mon. Weather Rev. 91, 99-164.

Smirnov, A., Shi, S., Celik, I., 2001. Random Flow Generation Technique for Large Eddy Simulations and ParticleDynamics Modeling. J. Fluids Eng. 123, 359. https://doi.org/10.1115/1.1369598

Tabor, G.R., Baba-Ahmadi, M.H., 2010. Inlet conditions for large eddy simulation: A review. Comput. Fluids 39, 553-567. https://doi.org/10.1016/J.COMPFLUID.2009.10.007

Tamura, T., 2008. Towards practical use of LES in wind engineering. J. Wind Eng. Ind. Aerodyn. 96, 1451-1471. https://doi.org/10.1016/j.jweia.2008.02.034

Tominaga, Y., Mochida, A., Yoshie, R., Kataoka, H., Nozu, T., Yoshikawa, M., Shirasawa, T., 2008. AIJ guidelines for practical applications of CFD to pedestrian wind environment around buildings. J. Wind Eng. Ind. Aerodyn. 96, 1749-1761. https://doi.org/10.1016/j.jweia.2008.02.058

Tominaga, Y., Stathopoulos, T., 2013. CFD simulation of near-field pollutant dispersion in the urban environment: A review of current modeling techniques. Atmos. Environ. 79, 716-730. https://doi.org/10.1016/j.atmosenv.2013.07.028

Tominaga, Y., Stathopoulos, T., 2012. CFD Modeling of Pollution Dispersion in Building Array: Evaluation of turbulent scalar flux modeling in RANS model using LES results. J. Wind Eng. Ind. Aerodyn. 104-106, 484491. https://doi.org/10.1016/j.jweia.2012.02.004

Tominaga, Y., Stathopoulos, T., 2011. CFD modeling of pollution dispersion in a street canyon: Comparison between LES and RANS. J. Wind Eng. Ind. Aerodyn. 99, 340-348. https://doi.org/10.1016/j.jweia.2010.12.005

Van Driest, E.R., 1956. On Turbulent Flow Near a Wall. J. Aeronaut. Sci. 23, 1007-1011.

VDI, 2005. VDI Guideline 3783/9, Environmental Meteorology-Prognostic Microscale Windfield ModelsEvaluation for Flow Around Buildings and Obstacles. Beuth Verlag,Berlin.

Venetsanos, A.G., Papanikolaou, E.A., Bartzis, J.G., 2010. The ADREA-HF CFD code for consequence assessment of hydrogen applications. Int. J. Hydrogen Energy 35, 3908-3918.

Vreman, A.W., 2004. An eddy-viscosity subgrid-scale model for turbulent shear flow: Algebraic theory and applications. Phys. Fluids 16, 3670-3681. https://doi.org/10.1063/1.1785131

Wang, H., Colvile, R.N., Pain, C., Aristodemou, E., ApSimon, H.M., 2011. Understanding peak pedestrian exposures due to traffic emissions within the urban environment. Transp. Res. Part D Transp. Environ. 16, 392-401. https://doi.org/10.1016/J.TRD.2011.03.002

Wang, W., Ng, E., Yuan, C., Raasch, S., 2017. Large-eddy simulations of ventilation for thermal comfort - A parametric study of generic urban configurations with perpendicular approaching winds. Urban Clim. 20, 202227. https://doi.org/10.1016/J.UCLIM.2017.04.007

Waterson, N.P., Deconinck, H., 2007. Design principles for bounded higher-order convection schemes - a unified approach. J. Comput. Phys. 224, 182-207.

Wu, X., 2017. Inflow Turbulence Generation Methods. Annu. Rev. Fluid Mech. 49, 23-49. https://doi.org/10.1146/annurev-fluid-010816-060322

Xie, Z.-T., 2011. Modelling Street-Scale Flow and Dispersion in Realistic Winds-Towards Coupling with Mesoscale Meteorological Models. Boundary-Layer Meteorol. 141, 53-75. https://doi.org/10.1007/s10546011-9629-х

Xie, Z.-T., Castro, I.P., 2009. Large-eddy simulation for flow and dispersion in urban streets. Atmos. Environ. 43, 2174-2185. https://doi.org/10.1016/J.ATMOSENV.2009.01.016

Xie, Z.-T., Castro, I.P., 2008. Efficient Generation of Inflow Conditions for Large Eddy Simulation of Street-Scale Flows. Flow, Turbul. Combust. 81, 449-470. https://doi.org/10.1007/s10494-008-9151-5

Xie, Z., Castro, I.P., 2006. LES and RANS for Turbulent Flow over Arrays of Wall-Mounted Obstacles. Flow, Turbul. Combust. 76, 291-312. https://doi.org/10.1007/s10494-006-9018-6 
Yoshie, R., Jiang, G., Shirasawa, T., Chung, J., 2011. CFD simulations of gas dispersion around high-rise building in non-isothermal boundary layer. J. Wind Eng. Ind. Aerodyn. 99, 279-288. https://doi.org/10.1016/J.JWEIA.2011.01.006

Yuan, C., Ng, E., Norford, L.K., 2014. Improving air quality in high-density cities by understanding the relationship between air pollutant dispersion and urban morphologies. Build. Environ. 71, 245-258. https://doi.org/10.1016/J.BUILDENV.2013.10.008

\section{Appendix A. Supplementary data}
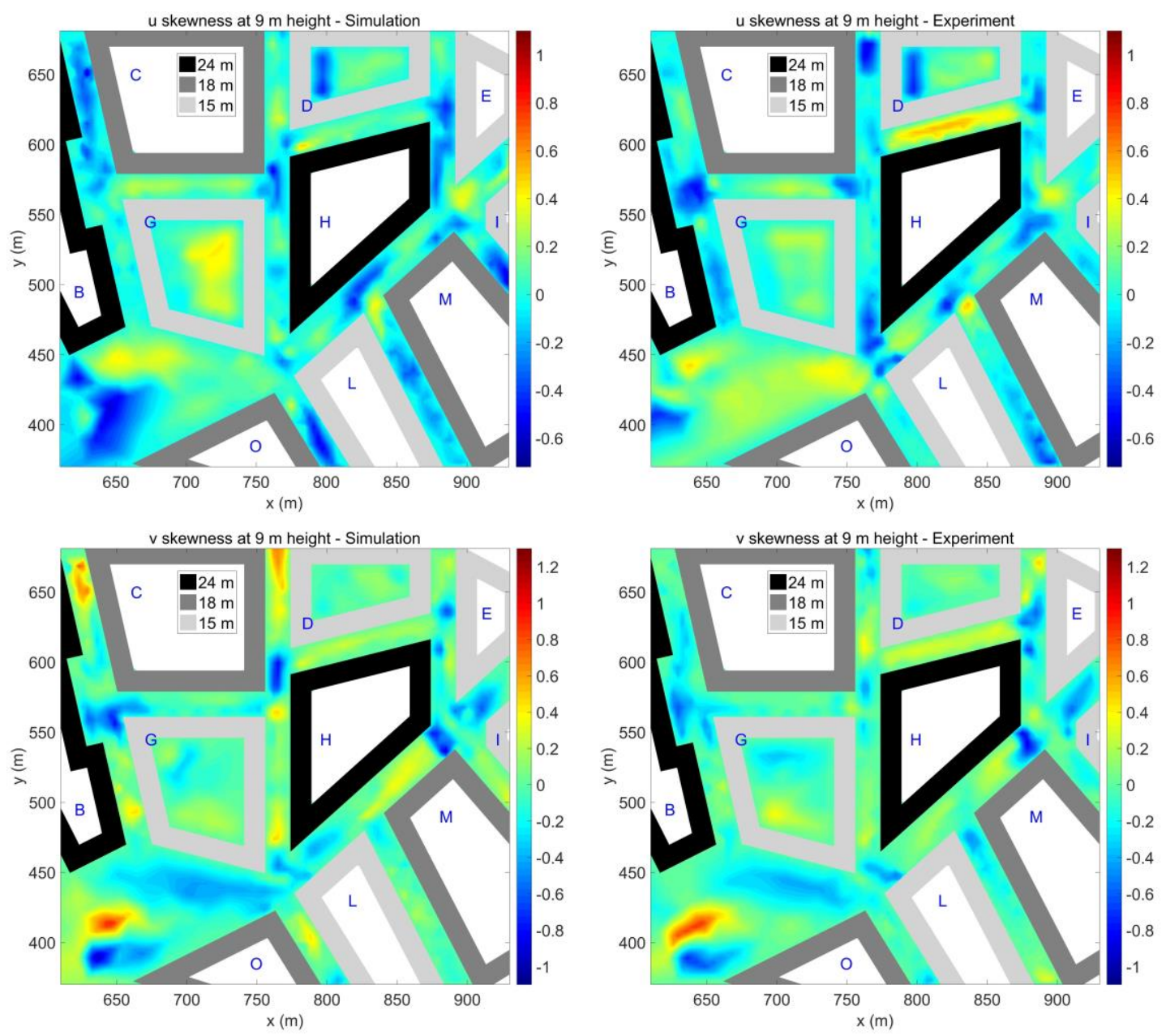

Figure A1. Skewness contours at $\mathrm{z}=9 \mathrm{~m}$ 

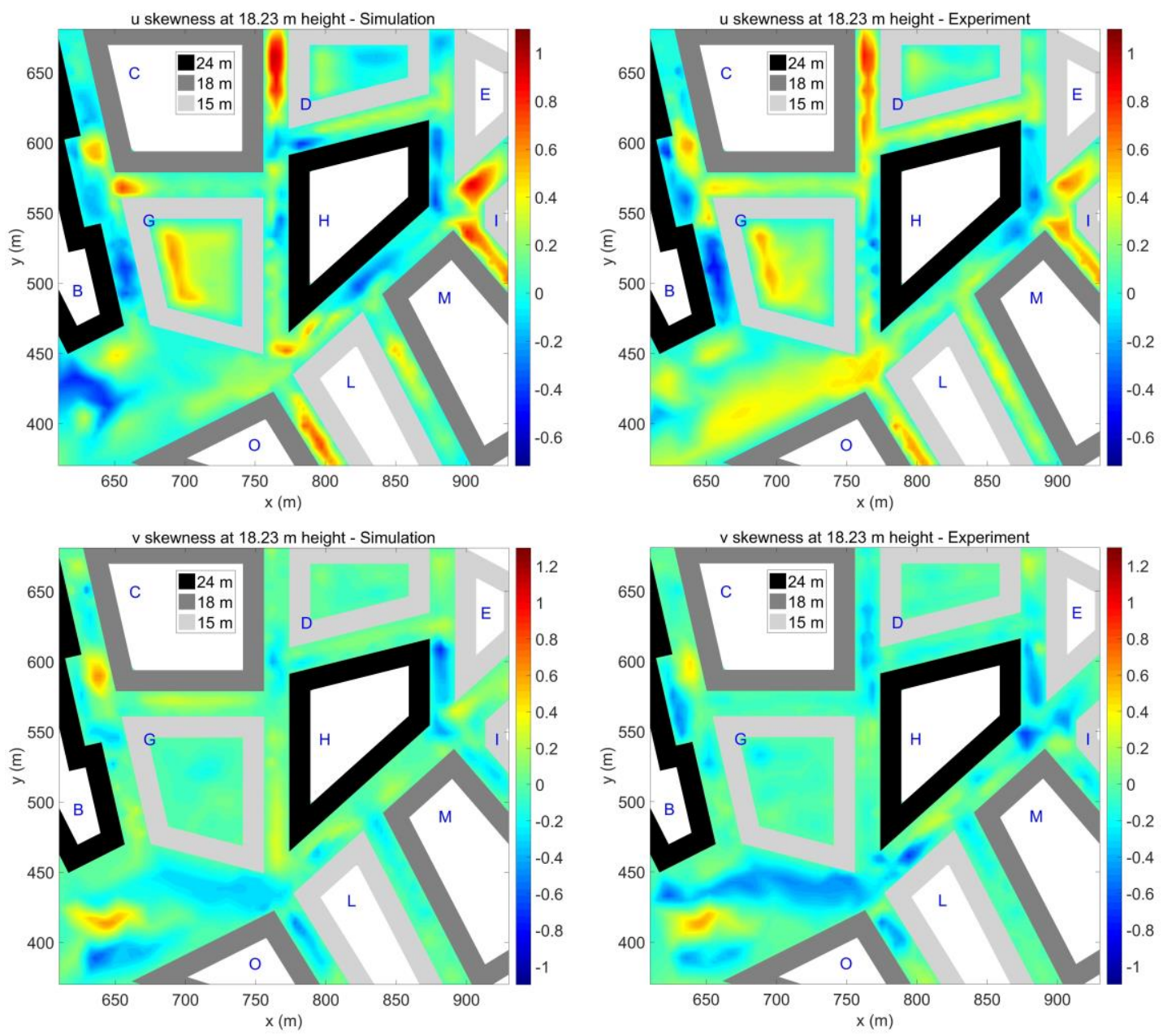

Figure A2. Skewness contours at $\mathrm{z}=18.23 \mathrm{~m}$
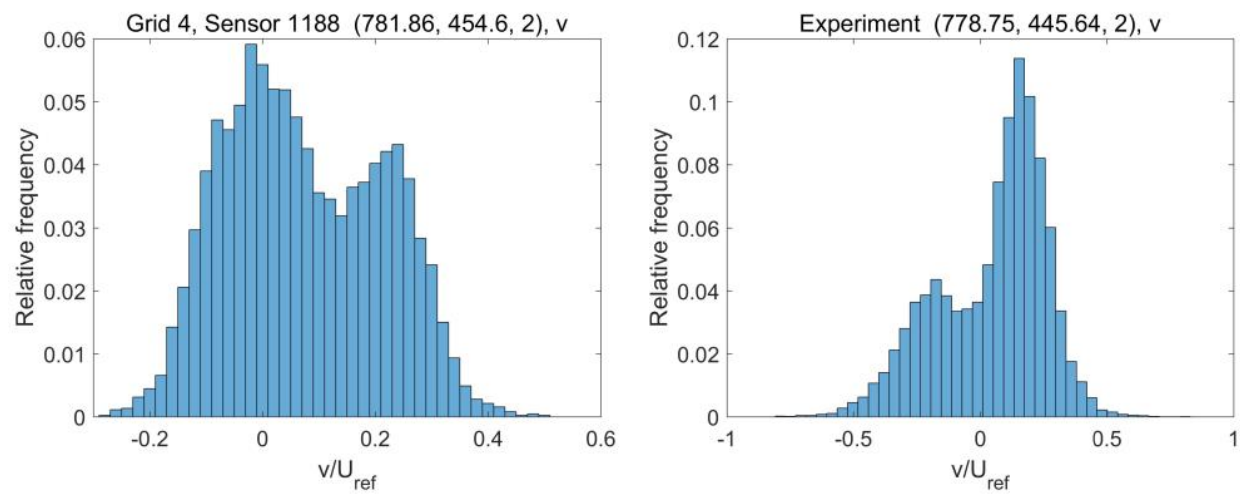

Figure A3. Frequency distribution for LES (Grid 4) and experiment at neighbouring points

\section{Videos:}

Scatter plots evolution for the streamwise velocity component as the grid is refined Scatter plots evolution for the lateral velocity component as the grid is refined 